\title{
DISEÑO ÓPTIMO MULTI-OBJETIVO DE EDIFICIOS DE CONCRETO REFORZADO USANDO ALGORITMOS GENÉTICOS
}

\author{
Edén Bojórquez Mora $^{(1)}$, Herian Leyva Madrigal ${ }^{(1)}$, Alfredo Reyes Salazar ${ }^{(1)}$, Eduardo Fernández \\ González $^{(1)}$, Juan Bojórquez Mora ${ }^{(1)}$, Jesús Leal Graciano ${ }^{(1)}$ y Juan Serrano Corona ${ }^{(1)}$
}

\begin{abstract}
RESUMEN
Se muestra el diseño sísmico óptimo de marcos estructurales de concreto reforzado mediante la aplicación de los algoritmos genéticos multi-objetivo, considerando dos objetivos de manera simultánea. El primer objetivo consiste en controlar la distorsión máxima de entrepiso y el segundo en minimizar el costo total estructural del marco. Para ello las estructuras se analizan representando el efecto sísmico a partir de fuerzas laterales. Para lograr un diseño sísmico satisfactorio se utiliza una búsqueda de soluciones basada en algoritmos evolutivos de optimización multi-objetivo denominada Non-Dominated Sorting Genetic Algorithm (NSGA-II). Los resultados obtenidos muestran que los algoritmos genéticos son una herramienta bastante útil para encontrar soluciones de problemas de optimización estructural, y que los diseños obtenidos son adecuados en términos de desempeño sísmico y economía.
\end{abstract}

Palabras clave: algoritmos genéticos; optimización multi-objetivo; marcos estructurales de concreto reforzado

\section{MULTI-OBJECTIVE OPTIMUM DESIGN OF R/C FRAMES USING GENETIC ALGORITHMS}

\begin{abstract}
The application of multi-objective genetic algorithms for the seismic design of reinforced concrete $(\mathrm{R} / \mathrm{C})$ frames is illustrated considering two objectives simultaneously. While the first objective of this study is to control the maximum inter-story drift, the second is to minimize the total cost of the frame. For this aim, the seismic effect is simulated through lateral forces for the analysis of the frames. To achieve a satisfactory seismic design, this work suggests the use of multi-objective evolutionary algorithms named Non-Dominated Sorting Genetic Algorithm (NSGA-II). The results indicate that genetic algorithm is a very useful tool for structural optimization; moreover, the designed frame buildings obtained are satisfactory in terms of seismic performance and economy.
\end{abstract}

Keywords: genetic algorithm; multi-objective optimization; R/C frames

\footnotetext{
Artículo recibido el 22 de mayo de 2017 y aprobado para su publicación el 17 de diciembre de 2018. Se aceptarán comentarios y/o discusiones hasta cinco meses después de su publicación.

(1) Facultad de Ingeniería, Universidad Autónoma de Sinaloa, Calzada de las Américas y Boulevard Universitarios S/N, Ciudad Universitaria, Culiacán, Sinaloa, México, C.P. 80040. eden@uas.edu.mx; herian 25@hotmail.com; reyes@uas.edu.mx; eddyf@uas.edu.mx; jbm_squall_cloud@hotmail.com; jesusleal@uas.edu.mx; juan.asc@ @otmail.com
} 


\section{INTRODUCCIÓN}

Con la aparición de las computadoras, la ingeniería estructural experimentó un cambio radical. En la actualidad se han desarrollado una gran cantidad de programas de análisis y diseño estructural obteniendo resultados más precisos y rápidos, generando diseños cada vez más económicos y seguros; además, surgieron nuevas áreas científicas como la Inteligencia Artificial y una de sus líneas más importantes son los Algoritmos Genéticos (Holland, 1975), que es una técnica de optimización que utiliza tácticas heurísticas basándose en los principios de selección natural de Charles Darwin enfocándose en la supervivencia, adaptación, cruzamiento y mutación de las especies a través del paso del tiempo. En la actualidad, este método se aplica en muchas áreas del conocimiento, teniendo mucho éxito por su fácil aplicación y excelentes resultados. Por ejemplo, en Ciencias Computacionales se han utilizado para el diseño de circuitos electrónicos (Lohn y Colombano, 1999); en Ciencias de la Tierra para el diseño de redes de distribución de agua de riego (Reca y Martínez, 2005); en Ciencias Financieras como método para la predicción de bancarrota (Back, et al., 1996) entre muchas otras aplicaciones. Combinando esta área del conocimiento con la ingeniería estructural, se puede desarrollar una técnica que sustituya la búsqueda de soluciones convencional, sin tomar en cuenta la habilidad del diseñador al dimensionar los elementos estructurales o el tiempo que tarda en obtener un resultado satisfactorio, con la cual se pretende obtener soluciones que cumplan con los criterios de diseño bajo cargas permanentes, variables y accidentales.

En el campo de la Ingeniería Estructural los algoritmos genéticos se han utilizado para el diseño de armaduras de acero (Deb y Gulati, 2001; Dede et al., 2011; Prendes, 2011), diseño óptimo de edificios de acero (Erbatur et al., 2000; Prendes et al., 2006), etc. Sin embargo, en la mayoría de los estudios mencionados, sólo se minimiza una función objetivo que generalmente se trata del peso de la estructura. Barraza, 2013 y Barraza et al., 2017 aplicaron los algoritmos genéticos con optimización multi-objetivo y diseñaron marcos de acero, los resultados mostraron una disminución considerable del peso estructural lo cual se refleja de manera positiva en el costo total de la estructura y la disminución de la diferencia entre la distorsión permitida y la distorsión máxima de entrepiso. Kaveh et al., (2015) aplicó esta herramienta para el diseño sísmico basado en el desempeño mediante análisis no lineales minimizando el costo inicial de la construcción y el costo de reparación. En ambos casos se observó que el potencial de los algoritmos genéticos es mejor para problemas de mayor complejidad.

Cuando se desea diseñar edificios de concreto reforzado (CR), existe una mayor cantidad de posibles soluciones en comparación con las edificaciones de acero, debido a la cantidad de combinaciones que se pueden generar en una sección con diferentes armados de acero longitudinal y de acero transversal. En el caso de la aplicación de algoritmos genéticos en estructuras de concreto, la mayoría de las investigaciones están enfocadas en el diseño de trabes (Coello et al., 1995; Leps y Sajnoha, 2003) y pocos para el diseño de marcos de concreto reforzado (Da Silva, 2001; Kripakaran et al., 2011). Por ejemplo, Babaei y Mollayi (2016), Serrano (2016) utilizaron la técnica evolutiva NSGA-II para diseñar marcos de concreto reforzado de 2 y 3 dimensiones respectivamente minimizando el costo total de la construcción y el desplazamiento máximo bajo cargas definidas. Por tal motivo, en este estudio se utilizan los algoritmos genéticos para diseñar marcos espaciales de concreto reforzado de 3, 6 y 9 niveles a través del método NSGA-II (Deb $e t$ $a l ., 2000)$. Las cargas sísmicas $F_{i}$ se calculan para cada marco tomando en cuenta las dimensiones de las secciones utilizadas. Además, como el concreto es un material frágil y se presentan grietas cuando se somete a cargas reversibles reduciendo su rigidez y resistencia, se considerarán las inercias agrietadas en trabes y columnas. A continuación, se proporciona una breve descripción de los algoritmos genéticos y la técnica NSGA-II, y se describe el procedimiento empleado para diseñar los edificios de concreto reforzado mediante dicha técnica. 


\section{Algoritmos genéticos}

Los Algoritmos Genéticos son una técnica de optimización que se basa en la Teoría de Selección Natural, donde los individuos que tienen mayor éxito para sobrevivir son los más adaptados al medio que los rodea y tienen una mayor probabilidad de generar descendientes. Esto significa que los genes de los individuos mejor adaptados se propagarán en sucesivas generaciones. La combinación de buenas características puede algunas veces producir "superindividuos" cuya adaptación es mejor que la de los demás descendientes, de esta manera las especies evolucionan obteniendo mejores características de adaptación y supervivencia (Holland, 1975; Goldberg, 1989; Kuri-Morales y Galaviz-Casas, 2002).

La mayoría de los algoritmos genéticos son variaciones del algoritmo genético simple (AG) propuesto por Goldberg (1989), el cual consiste en tres operadores genéticos básicos: reproducción, cruzamiento y mutación.

- Reproducción: La reproducción se basa en los mecanismos de selección de la teoría de Darwin; es decir, los individuos mejor adaptados sobreviven (Koza, 1992).

- Cruzamiento: El objetivo del cruzamiento es crear variaciones en las nuevas poblaciones de individuos, produciendo nuevas soluciones de cadenas que consisten en partes tomadas de otras cadenas de soluciones denominados padres.

- Mutación: La mutación introduce cambios aleatorios en la población de una generación; en general, la mutación puede ser benéfica ya que permite introducir diversidad en una población.

Después de que se utilizan estos tres operadores se genera una nueva población. Este procedimiento ha sido ampliamente utilizado como método de optimización en el diseño de estructuras de acero (Pezeshk et al., 2000), o en la selección de acelerogramas para análisis dinámico de estructuras (Bojórquez et al., 2013) con muy buenos resultados.

\section{Optimización multi-objetivo}

La optimización multi-objetivo o multi-criterio tiene la particular característica de que no existe una solución única que optimice simultáneamente todos los criterios de calidad (objetivos), sino un conjunto de soluciones válidas donde todas y cada una de ellas deben ser consideradas. Esta característica es consecuencia del conflicto de los atributos y da lugar al concepto de soluciones dominadas. La optimización multi-objetivo consiste en obtener un conjunto de soluciones que satisfagan diversas funciones. Existen diversos tipos de algoritmos multi-objetivo de entre los cuales sobresalen SPEA2, NSGA-II, MOMGA-II, PAES, entre otros. Con más de una función objetivo a optimizar, cambia la noción de óptimo, pues es muy raro que un solo individuo optimice simultáneamente todos los objetivos de un problema multi-objetivo. En consecuencia, se intenta encontrar un conjunto de soluciones que sean buenas entre todos los objetivos, obteniendo diversas soluciones óptimas. Una solución es óptima si no es dominada por ninguna otra. Al conjunto de individuos no dominados se le denomina Frontera de Pareto (Túpac, 2011).

Dominancia de Pareto: Para dos vectores de decisión: $\overrightarrow{x^{*}}, \overrightarrow{y^{*}} \in X$ se dice que $x^{*}$ domina a $y^{*}$ si y sólo si:

- La solución $\overrightarrow{x^{*}}$ no es peor que $\overrightarrow{y^{*}}$ en ninguno de sus objetivos. En caso de minimización la formula sería:

$$
f_{j}\left(\overrightarrow{x^{*}}\right) \leq f_{j}\left(\overrightarrow{y^{*}}\right), \forall j=1,2, \ldots M
$$


- La solución $\overrightarrow{x^{*}}$ es estrictamente mejor que la solución $\overrightarrow{y^{*}}$ en al menos un objetivo si

$f_{j}\left(\overrightarrow{x^{*}}\right)<f_{j}\left(\overrightarrow{y^{*}}\right)$, para al menos un $j \in 1,2, \ldots, M$

y se denota como $f\left(x^{*}\right)<f\left(y^{*}\right)$.

En la ejecución de un algoritmo multi-objetivo es importante tener una jerarquía a la hora de seleccionar los individuos sobrevivientes, por lo cual en cada generación se ejecuta un criterio de selección que se basa en separar a los individuos en fronteras en función de la no dominancia. Como se mencionó anteriormente existen diversas técnicas multi-objetivo, en este trabajo se utiliza la técnica NSGA-II (Deb, 2002). Además, también existe un criterio de selección entre dos individuos pertenecientes a la misma frontera conocida como Distancia de Crowding en la que se estima la densidad de las soluciones alrededor de una solución particular i, el algoritmo NSGA-II usa un procedimiento que toma la distancia promedio de dos soluciones para cada uno de sus objetivos como se observa en la figura 1.

La diferencia entre los valores de la función objetivo 1 de los individuos $i+1$ e $i$ - 1 , forman la longitud de la base del rectángulo que se forma del individuo $i$ con sus vecinos, mientras que la diferencia de la función objetivo 2 entre los mismos individuos, forman la longitud de la altura del rectángulo. Los individuos que se encuentran en los extremos de la frontera poseen un valor infinito.

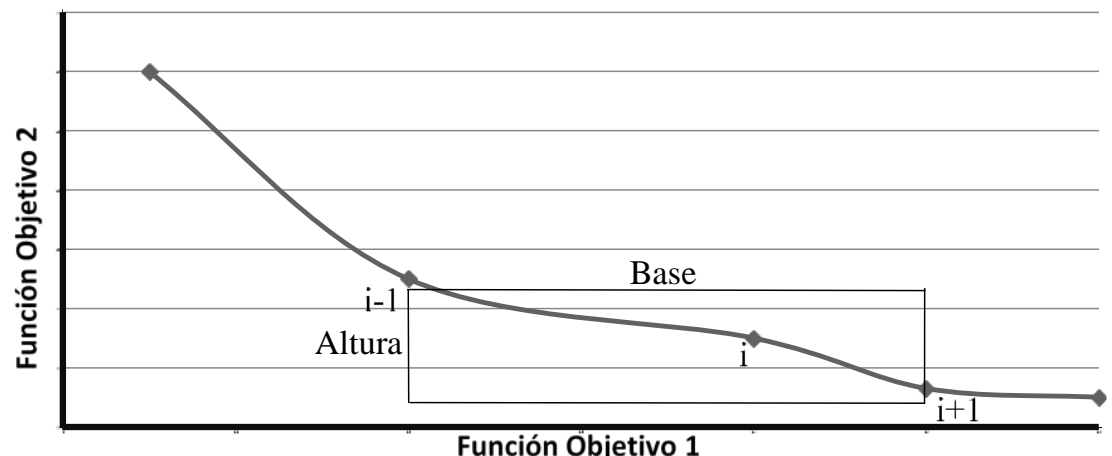

Figura 1. Representación gráfica de la Distancia de Crowding

El algoritmo utilizado para el cálculo de la medida de aglomeración (Distancia de Crowding) se describe a continuación:

1. Asigna a cada solución del conjunto una Distancia Crowding igual a cero $\left(d_{i}=0\right)$.

2. Para cada función objetivo $(m=1,2,3 \ldots)$, ordenar de forma creciente el conjunto o buscar el vector de índices ordenados: $I_{m}=\operatorname{sort}\left(F_{m},>\right)$.

3. Para cada función objetivo $(m=1,2,3, \ldots)$ asignar una distancia grande a las soluciones de los limites $\left(d_{I_{1}^{m}}=d_{I_{L}^{m}}=\infty\right)$ y para el resto de las soluciones asignar $(j=2$ a $l-1)$ :

$d_{I_{j}^{m}}=d_{I_{j}^{m}}+\frac{f_{m}^{\left(I_{j+1}^{m}\right)}-f_{m}^{\left(I_{j-1}^{m}\right)}}{f_{m}^{\max }-f_{m}^{\min }}$ 
donde $f_{m}$ es el valor de la función objetivo $m, f_{m}^{\max }$ y $f_{m}^{\min }$ son los valores máximos y mínimos de la función objetivo $m, I_{j}$ denota el índice de la solución $j$ de la lista ordenada e $I_{l}$ e $I_{L}$ los valores más altos y más bajos de la función objetivo. Se ordenan los individuos de la frontera de mayor a menor número de Distancia de Crowding, esto permite obtener una mejor distribución de los individuos.

\section{CRITERIOS DE DISEÑO}

Las acciones y criterios que se utilizaron para el diseño de los edificios de CR se tomaron de las Normas Técnicas Complementarias sobre criterios y acciones para el diseño estructural de las edificaciones y de las Normas Técnicas Complementarias para el Diseño por Sismo del Reglamento de Construcción del Distrito Federal Edición 2004 (RCDF, 2004).

\section{Cargas vivas}

Para la aplicación de las cargas vivas unitarias se deberá tomar en consideración las siguientes disposiciones:

a) La carga viva máxima $(\mathrm{Wm})$ se deberá emplear para diseño estructural por fuerzas gravitacionales y para calcular asentamientos inmediatos en suelos.

b) La carga instantánea $(\mathrm{Wa})$ se deberá usar para diseño sísmico y por viento y cuando se revisen distribuciones de carga más desfavorables que la uniformemente repartida sobre toda el área.

\section{Combinaciones de acciones y factores de carga}

La seguridad de una estructura deberá verificarse para el efecto combinado de todas las acciones que tengan una probabilidad no despreciable de ocurrir simultáneamente, considerándose dos categorías de combinaciones:

a) Para las combinaciones que incluyan acciones permanentes y acciones variables, se considerarán todas las acciones permanentes que actúen sobre la estructura y las distintas acciones variables. Para la combinación de carga muerta más carga viva, se empleará la intensidad máxima de la carga viva considerándola uniformemente repartida sobre toda el área. Cuando se trate de edificaciones del Grupo A, el factor de carga se tomará de 1.5, en caso contrario un factor de 1.4.

Combinación 1: $1.5 \mathrm{CM}+1.5 \mathrm{Wm}$

Combinación 1: $1.4 \mathrm{CM}+1.4 \mathrm{Wm}$

b) Para las combinaciones que incluyan acciones permanentes, variables y accidentales, se considerarán todas las acciones permanentes, las acciones variables con sus valores instantáneos y únicamente una acción accidental en cada combinación. En este estudio sólo se tomará en cuenta como acción accidental el sismo. Para este caso se tomará un factor de carga de 1.1 a todas las acciones que intervengan en la combinación.

Combinación 2: $1.1 \mathrm{CM}+1.1 \mathrm{Wa}+1.1$ SISMO 


\section{Criterios para diseño sísmico}

En este trabajo se utilizó el Método Estático Equivalente, el cual se puede utilizar para marcos simétricos no mayores que $30 \mathrm{~m}$ o marcos asimétricos no mayores que $20 \mathrm{~m}$. El método se basa en suponer un conjunto de fuerzas horizontales actuando sobre cada uno de los puntos donde están concentradas las masas de la estructura, cada fuerza se toma igual al peso de la masa correspondiente multiplicada por un coeficiente proporcional a la altura de la masa sobre el desplante. Para calcular las fuerzas horizontales que actúan sobre los marcos se utiliza la siguiente ecuación (RCDF, 2004):

$F_{i}=\frac{c}{Q} W_{i} h_{i} \frac{\sum W_{i}}{\sum W_{i} h_{i}} ; \quad \frac{c}{Q} \geq a_{0}$

donde $F_{i}$ es la fuerza horizontal que actúa sobre nivel $i, c$ es el coeficiente sísmico, $W_{i} h_{i}$ es el peso correspondiente al nivel $i$ multiplicado por su altura, $\sum W_{i}$ es la sumatoria de los pesos de todos los niveles, $\sum W_{i} h_{i}$ es la sumatoria de los pesos multiplicados por sus alturas correspondientes, $a_{0}$ es la ordenada espectral cuando $T=0$ y $Q$ es el factor de comportamiento sísmico.

\section{Revisión de desplazamientos laterales}

Los desplazamientos laterales de pisos consecutivos producidos por las cargas sísmicas no deberán de exceder 0.012 veces la diferencia de elevaciones como se explica en la sección 1.8 de las Normas Técnicas Complementarias para el Diseño por Sismo. El desplazamiento será el resultante del análisis con las fuerzas sísmicas reducidas, multiplicadas por el factor de comportamiento sísmico $Q$.

\section{Rigidez efectiva}

El concreto es un material con bajo nivel de elasticidad, esto se puede observar fácilmente si se somete un espécimen a esfuerzos de compresión crecientes hasta llevarlos a la falla, el comportamiento elástico se mantiene hasta una carga aplicada menor que $0.5 f^{\prime} c$, donde aparecen las primeras grietas disminuyendo la rigidez y elasticidad del material. En marcos de concreto reforzado bajo cargas sísmicas se presentan esfuerzos tanto de compresión como de tensión provocando agrietamientos en las secciones, pérdida de rigidez y de elasticidad. Para tomar en cuenta este fenómeno en el análisis sísmico, se reduce la rigidez de los elementos estructurales. Diversos investigadores han estudiado los efectos de la rigidez efectiva en marcos de concreto, Pique y Burgos (2008), concluyeron que es importante considerarla para obtener distorsiones reales; además, los momentos de diseño son menores a los valores que se obtuvieran si no se consideran secciones agrietadas por lo que el área de acero en el armado de las trabes puede disminuirse reduciendo el costo de la edificación.

Los reglamentos de construcción y algunos investigadores aplican la reducción en dos formas distintas: la primera es reducir en un porcentaje el momento de inercia gruesa de la sección $\left(\mathrm{I}_{\mathrm{g}}\right)$; y la segunda, reducir el valor total de la rigidez de la sección, en esta investigación se utilizaron los factores de reducción propuesto por las NTC-04. A continuación, se indican algunas propuestas para reducir la inercia o rigidez efectiva en la literatura o práctica de la ingeniería estructural. 
1. El Reglamento para Concreto Estructural ACI 318S-05 considera los siguientes factores de reducción de inercia (rigidez efectiva) para evaluar los desplazamientos laterales relativos de entrepiso:

\begin{tabular}{lcc}
\hline \multicolumn{1}{c}{ Secciones } & Inercia efectiva & Rigidez efectiva \\
\hline Trabes & $0.35 \mathrm{I}_{\mathrm{g}}$ & $\mathrm{Ec} \mathrm{I}_{\mathrm{e}}$ \\
Columnas & $0.70 \mathrm{I}_{\mathrm{g}}$ & $\mathrm{Ec} \mathrm{I}_{\mathrm{e}}$ \\
\hline
\end{tabular}

2. FEMA 356 considera:

\begin{tabular}{|c|c|c|}
\hline Secciones & Inercia gruesa & Rigidez efectiva \\
\hline Trabes & $\mathrm{I}_{\mathrm{g}}$ & $0.5 \mathrm{Ec} \mathrm{I}_{\mathrm{g}}$ \\
\hline $\begin{array}{l}\text { Columnas } \\
\mathrm{Pu}>0.5 \mathrm{f}^{\prime} \mathrm{c} \mathrm{Ag}\end{array}$ & $\mathrm{I}_{\mathrm{g}}$ & $0.7 \mathrm{Ec} \mathrm{I}_{\mathrm{g}}$ \\
\hline $\begin{array}{l}\text { Columnas } \\
\mathrm{Pu}<0.3 \mathrm{f}^{\prime} \mathrm{c} \mathrm{Ag}\end{array}$ & $\mathrm{I}_{\mathrm{g}}$ & $0.5 \mathrm{Ec} \mathrm{I}_{\mathrm{g}}$ \\
\hline
\end{tabular}

3. Factores de reducción de Paulay y Priestley (1992):

\begin{tabular}{lcc}
\hline \multicolumn{1}{c}{ Secciones } & Inercia agrietada & Rigidez efectiva \\
\hline $\begin{array}{l}\text { Trabes } \\
\text { Rectangulares } \\
\text { Columnas }\end{array}$ & $0.3-0.5 \mathrm{I}_{\mathrm{g}}$ & $\mathrm{Ec} \mathrm{I}_{\mathrm{e}}$ \\
$\mathrm{Pu}>0.5 \mathrm{f}^{\prime} \mathrm{c} \mathrm{Ag}$ & $0.7-0.9 \mathrm{I}_{\mathrm{g}}$ & $\mathrm{Ec} \mathrm{I}_{\mathrm{e}}$ \\
$\begin{array}{l}\mathrm{Columnas} \\
\mathrm{Pu}<0.2 \mathrm{f}^{\prime} \mathrm{c} \mathrm{Ag}\end{array}$ & $0.5-0.7 \mathrm{I}_{\mathrm{g}}$ & $\mathrm{Ec} \mathrm{I}_{\mathrm{e}}$ \\
\hline
\end{tabular}

4. Factores de reducción de Rivera-Vargas et al., (2014):

\begin{tabular}{lcc}
\hline \multicolumn{1}{c}{ Secciones } & Inercia agrietada & Rigidez efectiva \\
\hline $\begin{array}{l}\text { Trabes rectangulares } \\
\text { Columnas }\end{array}$ & $0.5 \mathrm{I}_{\mathrm{g}}$ & $\mathrm{Ec} \mathrm{I}_{\mathrm{e}}$ \\
$\mathrm{Pu} / \mathrm{f}^{\prime} \mathrm{c} \mathrm{Ag}=0.5$ & $1.0 \mathrm{I}_{\mathrm{g}}$ & $\mathrm{Ec} \mathrm{I}_{\mathrm{e}}$ \\
$\begin{array}{l}\mathrm{Columnas} \\
\mathrm{Pu} / \mathrm{f}^{\prime} \mathrm{c} \mathrm{Ag}=0.3\end{array}$ & $0.6 \mathrm{I}_{\mathrm{g}}$ & $\mathrm{Ec} \mathrm{I}_{\mathrm{e}}$ \\
\hline
\end{tabular}

5. Factores de reducción NTC-04:

\begin{tabular}{lcc}
\hline \multicolumn{1}{c}{ Secciones } & Inercia agrietada & Rigidez efectiva \\
\hline Trabes Rectangulares & $0.5 \mathrm{I}_{\mathrm{g}}$ & Ec $\mathrm{I}_{\mathrm{e}}$ \\
Columnas & $1 \mathrm{I}_{\mathrm{g}}$ & Ec $\mathrm{I}_{\mathrm{g}}$ \\
\hline
\end{tabular}




\section{ELEMENTOS ESTRUCTURALES}

Como elementos estructurales se tomaron en cuenta losas, trabes y columnas que se encargan de soportar las cargas vivas, muertas y accidentales que actúan sobre el marco. Para ello, se elaboraron bases de datos de secciones (Leyva, 2016), las cuales se utilizan en un programa de cómputo que analiza miles de combinaciones de marcos de CR con la finalidad de encontrar las soluciones óptimas utilizando la técnica de algoritmo genéticos.

\section{Losas}

Para la base de datos de las losas se propusieron únicamente peraltes de 10 a $25 \mathrm{~cm}$, con armado superior igual en ambas direcciones, y armado inferior igual en ambas direcciones con varillas corrugadas \#3 o \#4 y fy = $4200 \mathrm{~kg} / \mathrm{cm}^{2}$ así como una separación máxima de hasta $30 \mathrm{~cm}$ entre varillas.

\section{Trabes}

En este estudio, para las trabes se utilizaron secciones rectangulares con peraltes que varían desde $1.5 b$ hasta $2.2 b$ (donde $b$ es la base de la sección transversal) con dimensiones obtenidas a partir de múltiplos de $5 \mathrm{~cm}$ comenzando con una base de $20 \mathrm{~cm}$. Se propuso el mismo armado en parte inferior y superior utilizando varilla corrugada \#3 a \#8 con $f y=4200 \mathrm{~kg} / \mathrm{cm}^{2}$, estribos \#2 con $f y=2800 \mathrm{~kg} / \mathrm{cm}^{2}$ y varilla \#3.

\section{Columnas}

En este proyecto se utilizan únicamente columnas cuadradas que varían en su sección transversal desde 20 hasta $95 \mathrm{~cm}$ armadas con varilla corrugada \#3 a \#8 y \#10 con $f y=4200 \mathrm{~kg} / \mathrm{cm}^{2}$ y acero del $1 \%$ al $4 \%$. El armado de estribos se realiza con varilla \#3 y separaciones de $5 \mathrm{~cm}$ hasta la mitad de la separación máxima permitida.

\section{PRECIOS DE CONSTRUCCIÓN}

Para cada elemento estructural que se creó en la base de datos se calculó su costo por metro lineal o metro cuadrado según sea su caso. Se tomó en cuenta el costo de los materiales (concreto, varilla, cimbra) y el costo por mano de obra (colocación de cimbra, colado y armado).

Tabla 1. Costos de materiales utilizados

\begin{tabular}{lc}
\hline \multicolumn{1}{c}{ Concepto } & Costo \\
\hline Concreto $f^{\prime} c=250 \mathrm{~kg} / \mathrm{cm}^{2}, \mathrm{TMA}=3 / 4 ”$ & $\$ 1,850 / \mathrm{m}^{3}$ \\
Varilla \#2 & $\$ 4.55 / \mathrm{ml}$ \\
Varilla Corrugada \#3 & $\$ 7.74 / \mathrm{ml}$ \\
Varilla Corrugada \#4 & $\$ 13.82 / \mathrm{ml}$ \\
Varilla Corrugada \#5 & $\$ 21.60 / \mathrm{ml}$ \\
Varilla Corrugada \#6 & $\$ 31.09 / \mathrm{ml}$ \\
Varilla Corrugada \#7 & $\$ 42.30 / \mathrm{ml}$ \\
Varilla Corrugada \#8 & $\$ 55.29 / \mathrm{ml}$ \\
Varilla Corrugada \#10 & $\$ 86.28 / \mathrm{ml}$ \\
PT de Cimbra & $\$ 8.50 / 5 \mathrm{usos}=\$ 1.7 / \mathrm{PT}$ \\
\hline
\end{tabular}




\section{Costo de mano de obra}

Para calcular el costo de mano de obra se utilizaron los siguientes rendimientos y salarios:

Tabla 2. Salarios y rendimientos por categoría

\begin{tabular}{cccc}
\hline Categoría & Concepto & Rendimiento & Salario \\
\hline Albañil (2) & Colado & $1.5 \mathrm{~m}^{3} /$ jor & $\$ 1200$ \\
Fierrero (1) & Armado & $170 \mathrm{~kg} /$ jor & $\$ 460$ \\
Carpintero (1) & Cimbrado & $215 \mathrm{PT} / \mathrm{jor}$ & $\$ 400$ \\
\hline
\end{tabular}

\section{PROCEDIMIENTO PARA EL DISEÑO SÍSMICO DE EDIFICIOS DE CONCRETO REFORZADO USANDO NSGA-II}

A continuación, se presenta el procedimiento desarrollado para el diseño óptimo de edificios de CR usando NSGA-II:

1. Población inicial: la primera generación será formada aleatoriamente. Cada individuo corresponde a un marco de CR y estará compuesto de distintos elementos, un elemento puede ser una sección de viga, de columna con su área de refuerzo correspondiente o la losa, donde cada uno será representado por un código binario. Por ejemplo, el numero binario 0000000000 representa el primer elemento de la base de datos. Se propone un número total de individuos de la población en todas las generaciones, en este caso será de 200.

2. Parámetros de diseño: desde que la población es creada, se calculan las acciones permanentes, variables y accidentales, y se compararán con las fuerzas resistentes de los elementos estructurales, así como los desplazamientos permitidos por el reglamento de construcción.

3. Funciones objetivo: se busca obtener el marco estructural con mejor relación desempeño sísmicocosto tomando en cuenta los criterios de diseño estipulados en el RCDF. Es decir, una función que depende de las distorsiones máximas de entrepiso y una segunda función relacionada con el costo total de la estructura, las cuales serán penalizadas en función de no cumplir con los criterios de diseño (desplazamiento, resistencia, entre otros). Para obtener los exponentes de cada variable de penalización, los autores ejecutaron el programa varias veces, con la finalidad de modificarlos y evaluar sus resultados. Con los exponentes de las ecuaciones 9 y 10 se eliminan de forma rápida aquellos marcos que no cumplen con el peralte de la losa, y de forma moderada aquellos que no cumplen por resistencia o desplazamiento. Las funciones objetivo a minimizar son:

$$
\begin{aligned}
& F_{1}=D N * F p_{\text {losa }}{ }^{5} * F p_{d}^{3} * F p_{\text {Conexión }} * F p_{R^{10}}^{\frac{1}{10}} \\
& F_{2}=\operatorname{Costo}^{\frac{1}{3}} * F p_{\text {losa }}{ }^{5} * F p_{d}^{3} * F p_{\text {Conexión }} * F p_{R^{\frac{1}{10}}}
\end{aligned}
$$

donde $F_{1}$ es la función objetivo que depende de la distorsión máxima de entrepiso, $F_{2}$ es la función objetivo relacionada con el costo total de la estructura, $D N$ es la distorsión normalizada que se explica a continuación:

$D N=$ Distorsión normalizada. Mediante esta ecuación se busca obtener marcos con distorsiones máximas de entrepiso cercanas al límite estipulado por el RCDF (0.012). Lo anterior sirve también para minimizar el tamaño de las secciones y obtener resultados de construcciones lo más ligeras y económicas posibles. El valor mínimo que puede tener DN y $F_{l}$ es de 1, y ocurre cuando la distorsión 
máxima de entrepiso del marco es igual a la capacidad límite permitida por el reglamento (0.012), y cumple con todos los criterios de diseño. DN se calcula mediante las ecuaciones 10 y 11.

Si la distorsión es menor que el límite:

$$
D N=\frac{\text { Límite permitido }}{\text { Distorsión maxima }}
$$

En caso contrario:

$$
D N=\left(\frac{\text { Distorsión maxima }}{\text { Límite permitido }}\right)^{5}
$$

Los demás términos son variables de penalización que comienzan con un valor igual a uno, hasta que no cumplan con los siguientes criterios:

$F p_{\text {losa }}=$ Función de penalización de peralte de losa cuando es menor que el peralte mínimo:

$F p_{\text {losa }}=\left(1-\frac{\text { Peralte losa }}{\text { Peralte mínimo }}\right)$

Cuando la losa es 2 centímetros mayor que el peralte mínimo:

$F p_{\text {losa }}=\left(1-\frac{\text { Peralte mínimo }}{\text { Peralte losa }}\right)$

$F p_{d}=$ Función de penalización por desplazamiento de trabes y distorsiones de entrepiso:

$F p_{d}=\sum_{i=1}^{\# \text { barras }}\left(1-\frac{\text { limite }}{\text { desplazamiento }}\right)$

$F p_{\text {Conexión }}=$ Función de penalización en conexiones trabe-columna, cuando la base de la trabe es mayor que la base de la columna, o también cuando las dimensiones de la columna de los niveles superiores son mayores que la de las columnas inferiores:

$F p_{\text {Conexión }}=\sum_{i=1}^{\# \text { conexiones }}($ dim. mayor - dim. menor $)$

$F p_{R}=$ Factor de penalización por resistencia, si la barra o losa no resiste los elementos mecánicos que actúan sobre sí mismo, éste se penaliza de la siguiente forma:

$F p_{R}=\sum_{i=1}^{\# \text { barras }}\left(1-\frac{\text { Resistencia }}{\text { Fuerza Máxima }}\right)$

4. Selección: en este paso se seleccionan los 100 marcos (individuos) con las mejores funciones objetivo separándolos en capas o fronteras de conjuntos no dominados como se explicó en el Capítulo anterior. Estos marcos de cruzarán para obtener los individuos restantes de la nueva generación.

5. Cruzamiento: se considera cruzamiento en un solo punto. Consiste en combinar el código binario de 2 individuos (marcos) específicos para mezclar sus genes (características) y formar nuevos 
individuos, se utilizó una probabilidad de cruzamiento de 100\%. Para determinar los padres se utilizó el Torneo Binario (Deb, 2002).

6. Mutación: se utiliza para garantizar la diversidad de marcos obtenidos en cada generación. Se aplica para todas las generaciones y consiste en el cambio de un bit específico del código de un individuo. Se utilizó una probabilidad de mutación de 5\%.

7. Nueva Generación: después de todo el proceso evolutivo, se obtiene una nueva generación, y el proceso regresa al paso 2 para comenzar una nueva generación hasta completar un determinado número de generaciones $\mathrm{N}$, o se cumpla el fenómeno de convergencia.

Para definir la Frontera de Pareto, en cada modelo estudiado se deberá ejecutar el algoritmo varias veces hasta obtener un conjunto de soluciones no dominadas.

\section{EJEMPLOS DE DISEÑO ESTRUCTURAL}

Para esta investigación se propuso diseñar 3 marcos tridimensionales de CR simétricos en ambas direcciones ortogonales, 2 de los cuales cuentan con 3 crujías de 7 metros de longitud en ambas direcciones, y tienen 3 y 6 niveles con alturas de 3.5 metros para cada entrepiso. El otro marco se propuso de 9 niveles, al igual que los anteriores, cuenta con 3 crujías en ambas direcciones, pero con longitudes de crujía de 5 metros y una altura de entrepiso de 3 metros. Las características de los edificios a diseñar se muestran en las figuras 2 a 4 . Cabe mencionar que se propuso utilizar una columna y una trabe por cada 3 niveles; además, de una losa para todo el marco. En la tabla 3 se muestra el total de elementos (o secciones) distintos que se desea obtener para cada modelo estudiado.

Tabla 3. Número de elementos distintos utilizados en los modelos estudiados

\begin{tabular}{ccccc}
\hline Modelo & Trabe & Columna & Losa & Elementos \\
\hline Modelo CR-3 & 1 & 1 & 1 & 3 \\
Modelo CR-6 & 2 & 2 & 1 & 5 \\
Modelo CR-9 & 3 & 3 & 1 & 7 \\
\hline
\end{tabular}

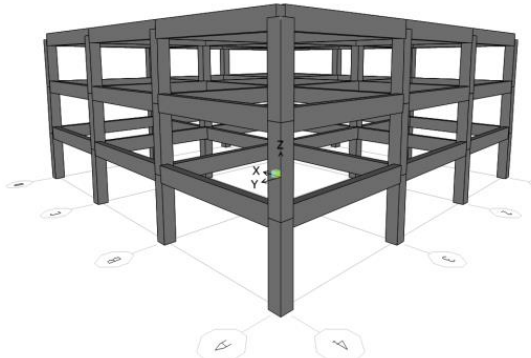

Figura 2. Modelo CR-3, marco de 3 niveles con 3 crujías en ambas direcciones de $7 \mathrm{~m}$ de longitud de crujía 


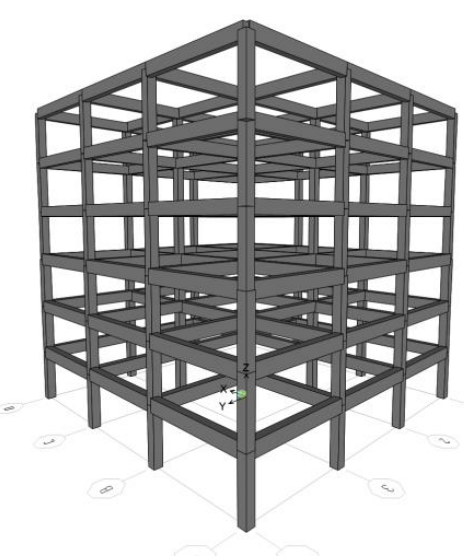

Figura 3. Modelo CR-6, marco de 6 niveles con 3 crujías en ambas direcciones de $7 \mathrm{~m}$ de longitud de crujía

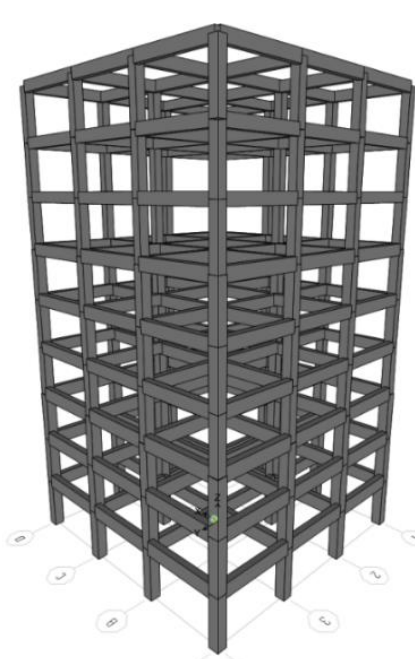

Figura 4. Modelo CR-9, marco de 9 niveles con crujías en ambas direcciones de $5 \mathrm{~m}$ de longitud de crujía

\section{RESULTADOS}

Se busca obtener un conjunto de resultados compuestos por las mejores soluciones al problema; es decir, encontrar la Frontera de Pareto. Para esto, los autores desarrollaron un software de computadora, que se ejecuta varias veces para obtener el conjunto de soluciones no dominadas.

El software elaborado utiliza la técnica de algoritmos genéticos, secciones de las bases de datos de elementos de concreto reforzado creadas, y para el diseño se tomaron en cuenta los siguientes valores:

Individuos por generación: 200

Número de generaciones: 100

Zona donde se supone ubicadas las estructuras: IIIb

Factor de comportamiento sísmico Q: 3

Tipo de edificación: B (oficinas) 


\section{Resultados para el modelo CR-3}

El comportamiento del programa fue adecuado, con el paso de las generaciones, las funciones objetivo fueron disminuyendo, principalmente el costo $\left(F_{2}\right)$. Se observó que primero encontraba la losa más económica, esto se debe a que la losa influye en poco más del $50 \%$ en el costo estructural total, después seleccionaba la columna y al final la trabe.

Para obtener una visión de lo mencionado anteriormente, en la figura 5 se muestran los valores de $F_{2}$ de todos los individuos creados en cada generación, donde se observa que va disminuyendo hasta alcanzar el más económico, lo mismo sucede para la función $F_{1}$.

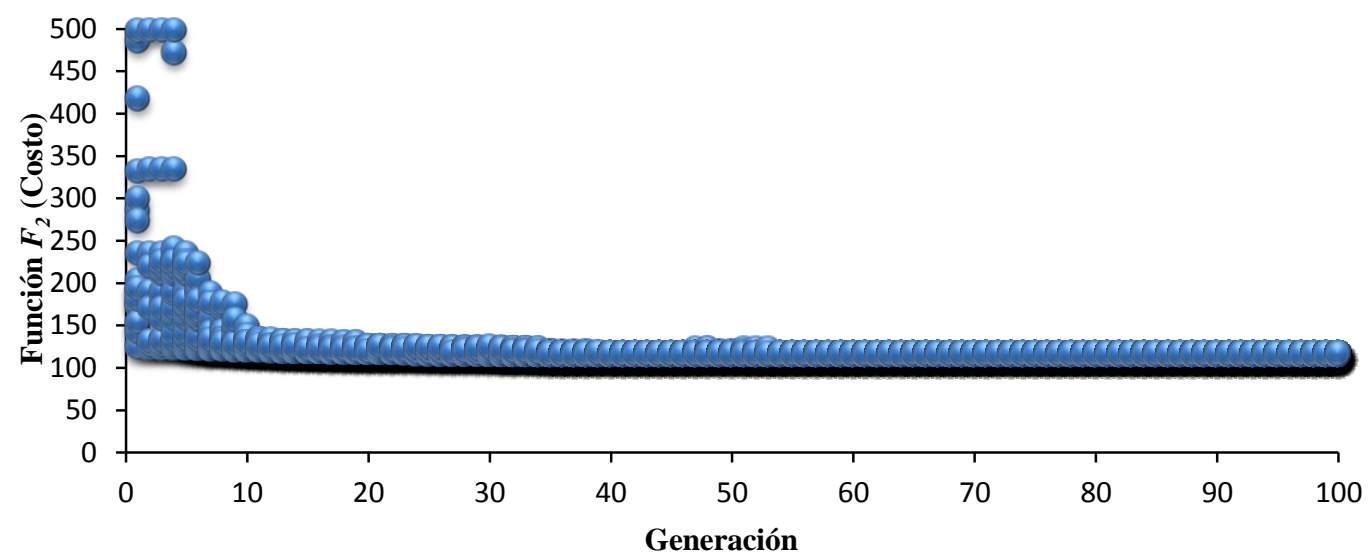

Figura 5. Evolución de la función objetivo $F_{2}$ en la primera ejecución del programa para el modelo CR-3

Los mejores resultados obtenidos se muestran en la tabla 4. Nótese que la distorsión máxima de entrepiso para las mejores estructuras obtenidas es muy cercana a 0.012; es decir, los marcos espaciales de CR desarrollan toda su capacidad de deformación, lo cual maximiza su eficiencia y reduce el costo considerablemente. Adicionalmente, la figura 6 compara las funciones objetivos obtenidas de la múltiple ejecución del algoritmo NSGA-II, donde se observa claramente la Frontera de Pareto (frontera 1) con 2 individuos.

Tabla 4. Mejores resultados para el modelo CR-3

\begin{tabular}{ccccc}
\hline Individuo & $F_{1}$ & $F_{2}$ & $\begin{array}{c}\text { Distorsión } \\
\text { máxima de } \\
\text { entrepiso }\end{array}$ & $\begin{array}{c}\text { Costo total } \\
\text { estructural }\end{array}$ \\
\hline 1 & 1.015 & 125.664 & 0.0118227 & $\$ 1,984,415.63$ \\
2 & 1.001 & 127.878 & 0.0119880 & $\$ 2,091,161.17$ \\
3 & 1.098 & 125.879 & 0.0109290 & $\$ 1,994,618.54$ \\
4 & 1.072 & 126.395 & 0.0111940 & $\$ 2,019,248.10$ \\
5 & 1.056 & 126.866 & 0.0113636 & $\$ 2,041,905.98$ \\
6 & 1.008 & 128.779 & 0.0119048 & $\$ 2,135,674.91$ \\
\hline
\end{tabular}




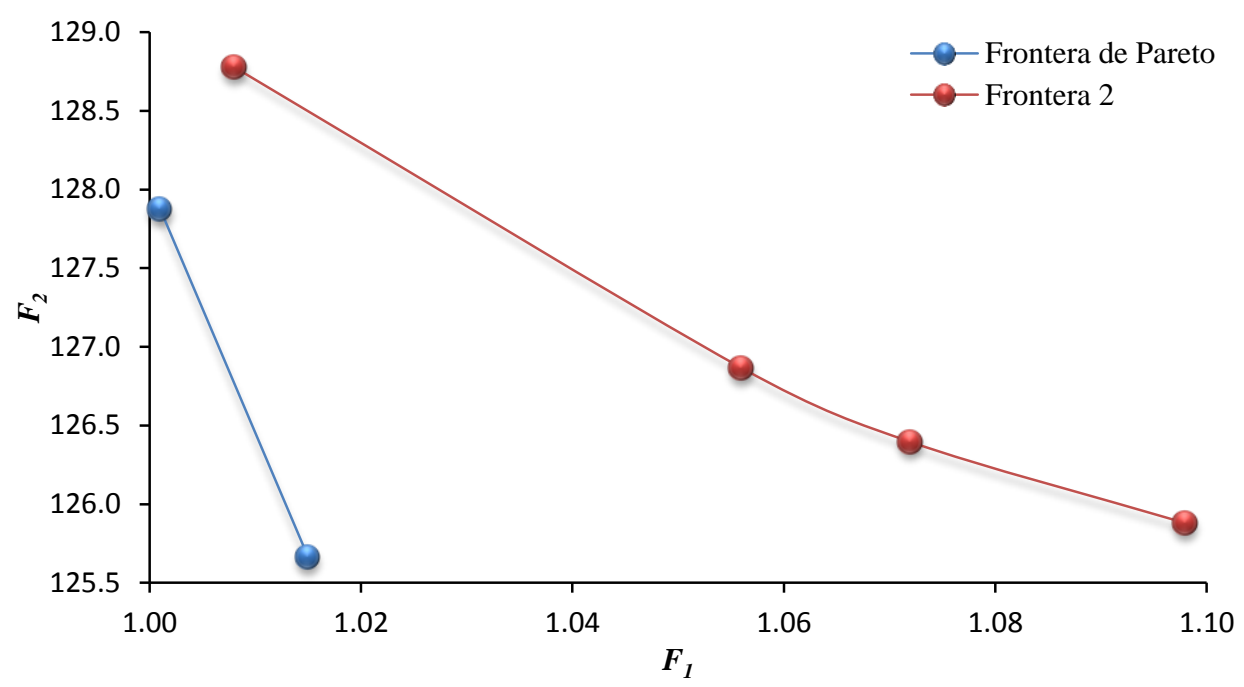

Figura 6. Funciones objetivo $F_{1}$ y $F_{2}$ de los mejores resultados del modelo CR-3

La Frontera de Pareto solo incluye 2 individuos por tener valores de $F_{l}$ muy pequeños y dominar por ello a los demás individuos. Aunque el resto de los individuos también son buenos resultados siendo más económicos que el individuo 2 y con menores distorsiones máximas de entrepiso. Por otro lado, la función objetivo $F_{l}$ se enfoca en hacer que los marcos sufran desplazamientos cercanos al límite permisible de acuerdo al RCDF. La siguiente figura compara los valores de distorsión máxima de entrepiso y costos reales. Los resultados sugieren que la diferencia entre las distorsiones máximas de entrepiso es mínima, mientras que en el costo total de los individuos de la Frontera de Pareto (frontera 1) la diferencia es de \$106,745.54. Como se mencionó anteriormente, el costo de la losa influye mucho en el costo total del marco, por lo que la diferencia entre los costos depende de las secciones de trabes y columnas. Para observar con mayor detalle las diferencias entre los marcos se presentan los costos y dimensiones de cada elemento estructural y cada marco (ver tabla 5). El costo de las secciones también depende del armado, que se representan de forma ilustrativa en las figuras 8 a 10 .

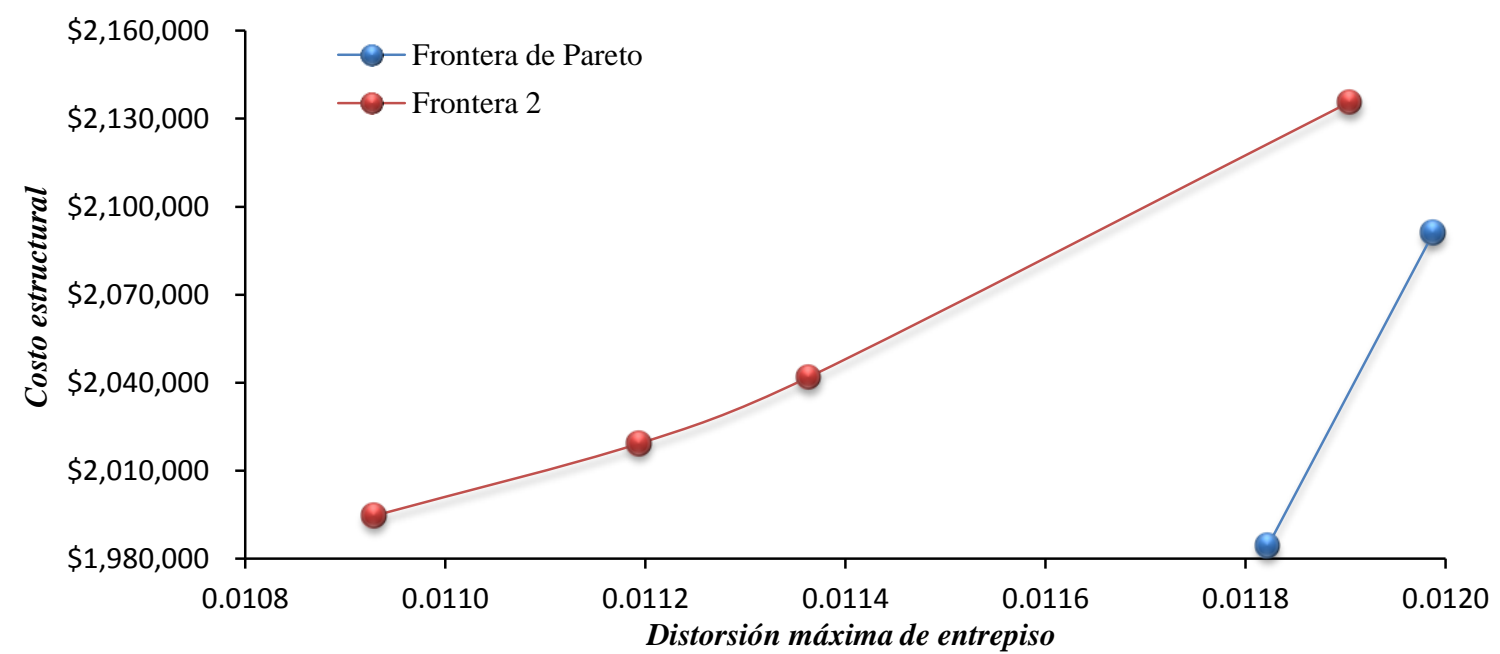

Figura 7. Valores reales de costo total estructural y distorsión máxima de entrepiso para los mejores resultados del modelo CR-3 
Tabla 5. Dimensiones y costos de las secciones de cada individuo (modelo CR-3)

\begin{tabular}{ccccccc}
\hline \multirow{2}{*}{ Individuo } & \multicolumn{2}{c}{ Secciones $(\mathrm{cm} \times \mathrm{cm})$} & \multicolumn{3}{c}{ Costo $\left(\$ / \mathrm{ml} \mathrm{o} \$ / \mathrm{m}^{2}\right)$} \\
\cline { 2 - 7 } & Columna & Trabe & Losa & Columna & Trabe & Losa \\
\hline 1 & $55 \times 55$ & $35 \times 65$ & 18 & $\$ 1,369.04$ & $\$ 1,268.68$ & $\$ 782.12$ \\
2 & $60 \times 60$ & $35 \times 60$ & 18 & $\$ 1,765.58$ & $\$ 1,346.17$ & $\$ 782.12$ \\
\hline
\end{tabular}

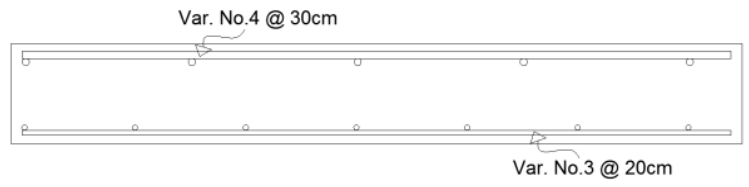

Figura 8. Losas de todos los individuos de la Frontera de Pareto para el modelo CR-3

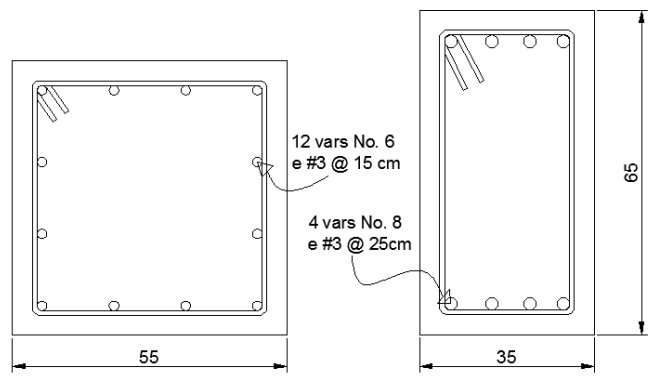

Figura 9. Armado de trabes y columnas para el individuo 1

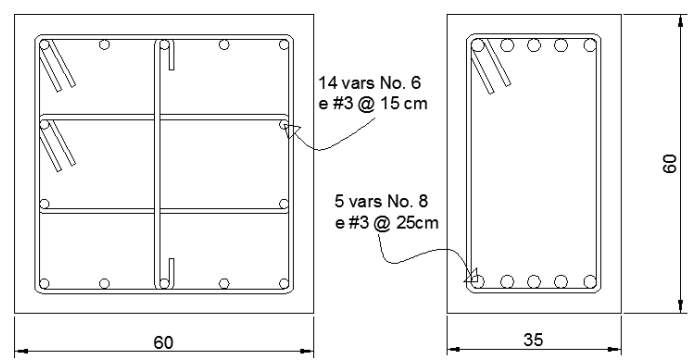

Figura 10. Armado de trabes y columnas para el individuo 2

\section{Resultados para el modelo CR-6}

El comportamiento del programa de cómputo fue similar al del modelo anterior. En la tabla 6 se muestran los 6 mejores individuos resultantes en la ejecución del programa. En esta ocasión, la Frontera de Pareto está definida por 3 individuos con costos totales estructurales muy cercanos entre ellos. Los valores de distorsión máxima de entrepiso en los individuos de la Frontera de Pareto varían entre 0.011 y 0.012, y como se mencionó los costos están muy cercanos entre sí, la diferencia mayor es de $\$ 78,105.67$ (ver figuras 11 y 12). Al igual que los resultados del modelo anterior, todos los individuos resultaron con la misma losa y trabe 1 (ver tabla 7), por lo tanto, la diferencia de costos se debe al resto de los elementos estructurales. Las trabes de los niveles inferiores resultaron con peraltes muy grandes, de hasta $100 \mathrm{~cm}$, indicando que para este modelo influye mucho la rigidez efectiva que se toma en cuenta. El tomar en cuenta solo un porcentaje de la inercia gruesa de las trabes en la rigidez total del marco para el análisis sísmico afecta considerablemente las demandas de distorsiones máximas de entrepiso. Lo anterior se ve claramente en los 
resultados. Las secciones resultantes de cada individuo para cada elemento estructural son las que se muestran en las figuras 13-16.

Tabla 6. Mejores resultados para el modelo CR-6

\begin{tabular}{ccccc}
\hline Individuo & $F_{1}$ & $F_{2}$ & $\begin{array}{c}\text { Distorsión } \\
\text { máxima de } \\
\text { entrepiso }\end{array}$ & $\begin{array}{c}\text { Costo total } \\
\text { estructural }\end{array}$ \\
\hline 1 & 1.087 & 170.205 & 0.0110396 & $\$ 4,930,794.94$ \\
2 & 1.049 & 170.762 & 0.0114395 & $\$ 4,979,379.08$ \\
3 & 1.001 & 171.099 & 0.0119880 & $\$ 5,008,900.61$ \\
4 & 1.049 & 171.056 & 0.0114395 & $\$ 5,005,125.10$ \\
5 & 1.015 & 172.063 & 0.0118227 & $\$ 5,094,041.42$ \\
6 & 1.001 & 172.286 & 0.0119880 & $\$ 5,113,873.30$ \\
\hline
\end{tabular}

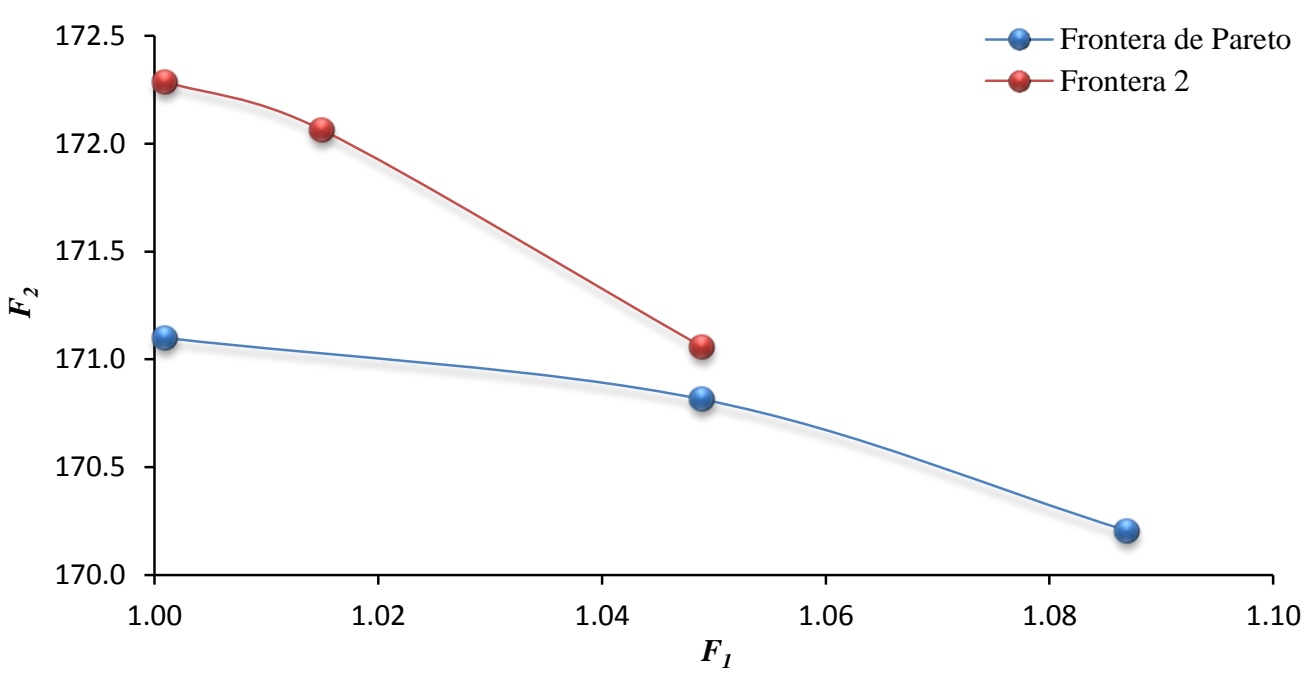

Figura 11. Funciones objetivo $F_{1}$ y $F_{2}$ de los mejores resultados del modelo CR-6

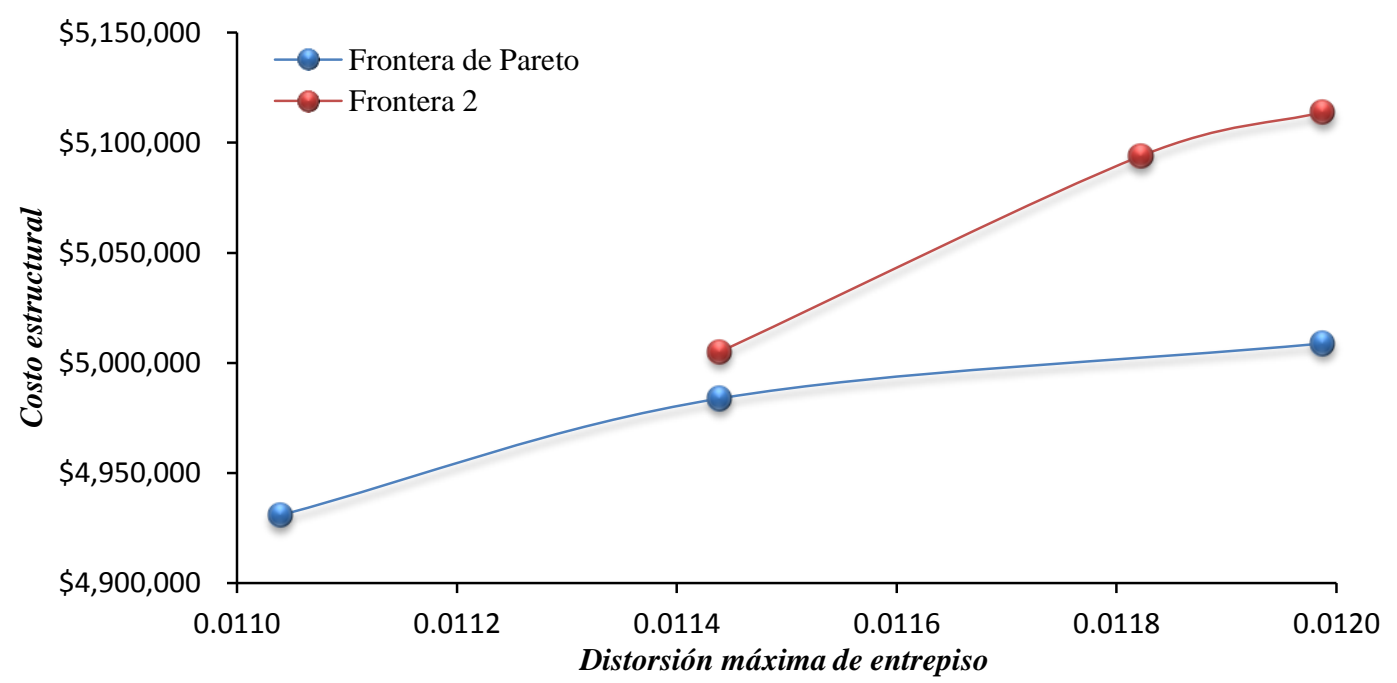

Figura 12. Valores reales de costo total y distorsión máxima para los mejores resultados del modelo CR-6 
Tabla 7. Dimensiones y costos de las secciones de cada individuo

\begin{tabular}{|c|c|c|c|c|}
\hline \multicolumn{2}{|c|}{ Individuo } & 1 & 2 & 3 \\
\hline \multirow{5}{*}{ 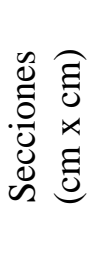 } & Columna 1 & $65 \times 65$ & $65 \times 65$ & $65 \times 65$ \\
\hline & Columna 2 & $65 \times 65$ & $60 \times 60$ & $55 \times 55$ \\
\hline & Trabe 1 & $45 \times 100$ & $45 \times 100$ & $45 \times 100$ \\
\hline & Trabe 2 & $35 \times 75$ & $45 \times 75$ & $40 \times 85$ \\
\hline & Losa & 18 & 18 & 18 \\
\hline \multirow{5}{*}{ 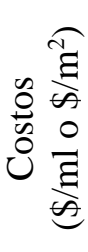 } & Columna 1 & $\$ 2,448.24$ & $\$ 2,103.77$ & $\$ 2,130.99$ \\
\hline & Columna 2 & $\$ 2,024.98$ & $\$ 1,831.74$ & $\$ 1,738.54$ \\
\hline & Trabe 1 & $\$ 2,349.04$ & $\$ 2,349.04$ & $\$ 2,349.04$ \\
\hline & Trabe 2 & $\$ 1,510.02$ & $\$ 1,787.80$ & $\$ 1,870.42$ \\
\hline & Losa & $\$ 782.12$ & $\$ 782.12$ & $\$ 782.12$ \\
\hline
\end{tabular}

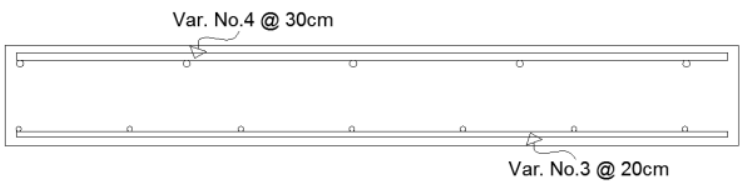

Figura 13. Losas de todos los individuos de la Frontera de Pareto para el modelo CR-6
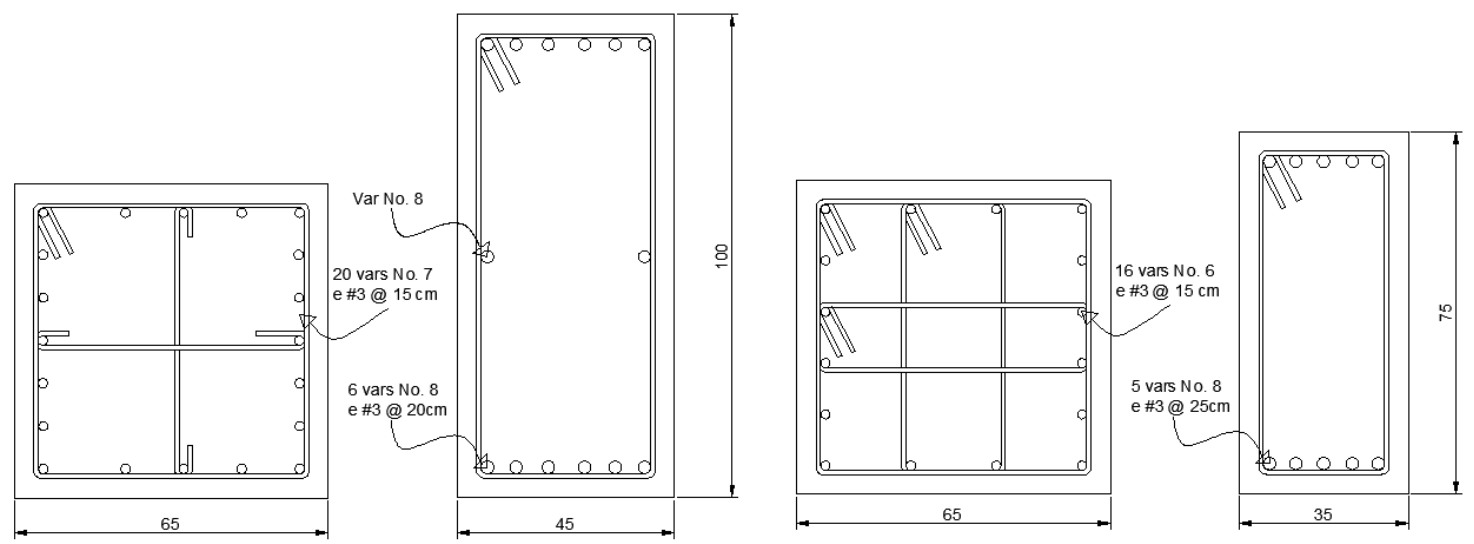

Figura 14. Armado de trabes y columnas para el individuo 1

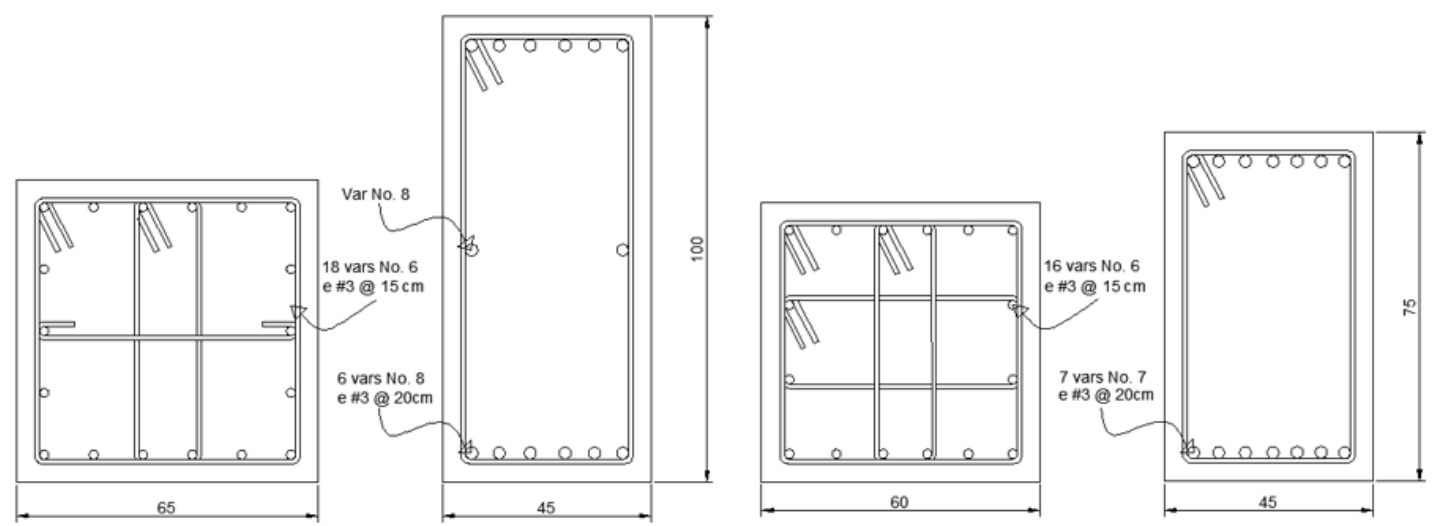

Figura 15. Armado de trabes y columnas para el individuo 2 


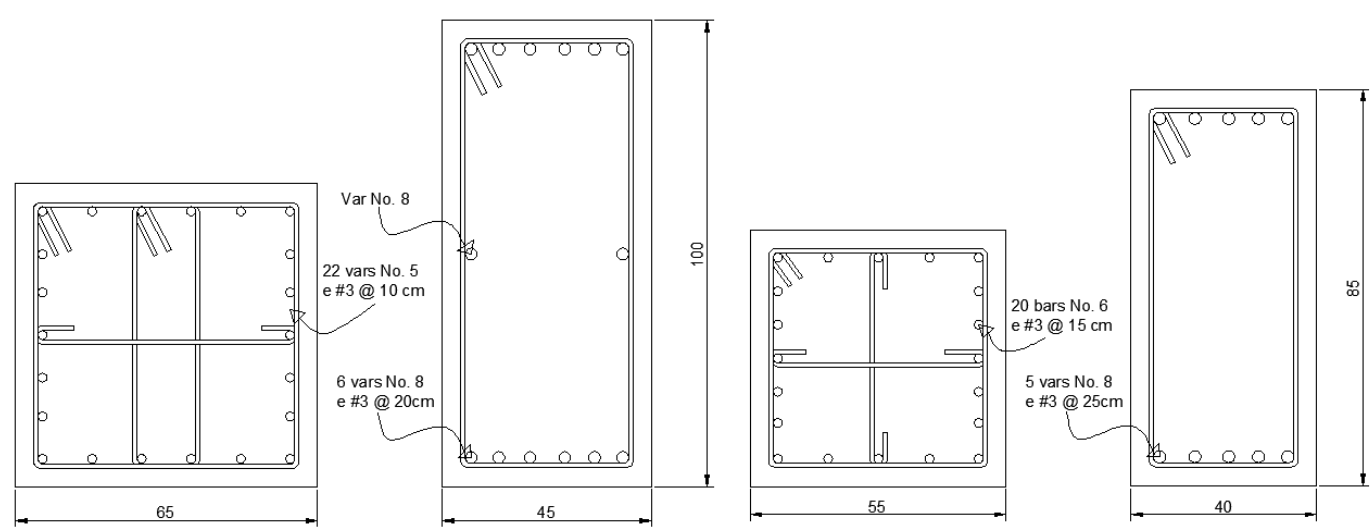

Figura 16. Armado de trabes y columnas para el individuo 3

\section{Resultados para el modelo CR-9}

Para este modelo se busca obtener un total de 7 elementos por individuo: 3 columnas, 3 trabes y una losa, por lo que existe una gran diversidad de posibles soluciones y resulta difícil que todos los marcos converjan a secciones similares. La tabla 8 indica los mejores resultados en términos de las funciones objetivo asociadas a costo y distorsión máxima de entrepiso. En los resultados se observa que la Frontera de Pareto está definida por 5 individuos (ver figura 17). Se observa que los valores de las funciones objetivo $F_{2}$ son muy cercanos entre sí para los individuos resultantes; además, existe una diferencia máxima entre los individuos que forman la Frontera de Pareto de $\$ 73,777.15$ como se ilustra en la tabla 8 y figura 18 que compara los resultados en términos de costo y desempeño estructural (distorsión máxima de entrepiso). Aunque los resultaron fueron satisfactorios, con el objetivo de definir de forma más adecuada la Frontera de Pareto, es deseable utilizar un mayor número de individuos por generación y un mayor número de generaciones para analizar más combinaciones y encontrar mejores resultados.

Tabla 8. Mejores resultados para el modelo CR-9

\begin{tabular}{ccccc}
\hline Individuo & $F_{1}$ & $F_{2}$ & Distorsión & Costo \\
\hline 1 & 1.025 & 150.539 & 0.01170732 & $\$ 3,411,513.40$ \\
2 & 1.023 & 150.542 & 0.01173021 & $\$ 3,411,717.35$ \\
3 & 1.009 & 150.729 & 0.01189296 & $\$ 3,424,508.25$ \\
4 & 1.006 & 151.029 & 0.01192843 & $\$ 3,444,997.81$ \\
5 & 1.001 & 151.679 & 0.01198801 & $\$ 3,489,669.75$ \\
6 & 1.009 & 151.157 & 0.01189296 & $\$ 3,453,701.44$ \\
7 & 1.006 & 153.618 & 0.01192843 & $\$ 3,625,152.83$ \\
\hline
\end{tabular}




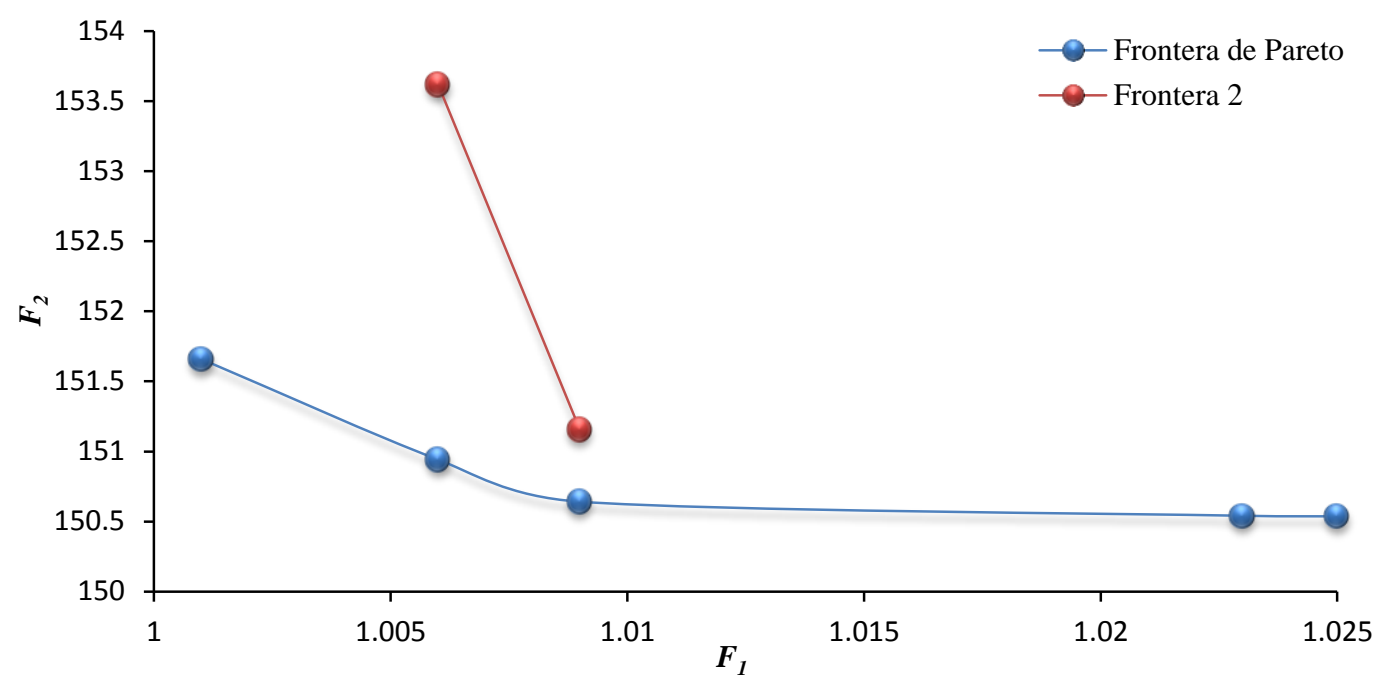

Figura 17. Funciones objetivo $F_{1}$ y $F_{2}$ de los mejores resultados del modelo CR-9

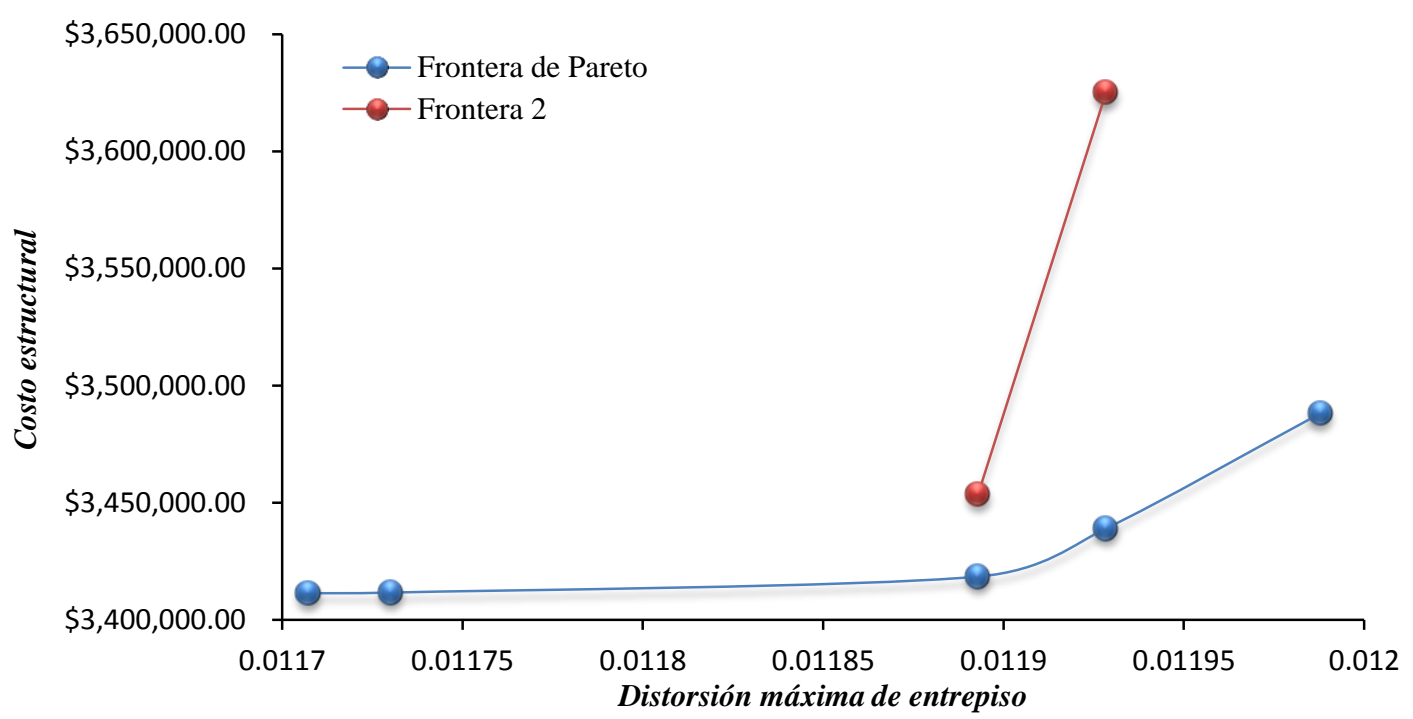

Figura 18. Valores reales de costo total y distorsión máxima para los mejores resultados del modelo CR-9

Como se explicó anteriormente, en este caso era difícil la convergencia a secciones similares y que se repitiera en todos los individuos por la cantidad de elementos y combinaciones que existen. Sin embargo, las dimensiones y costos de los elementos estructurales son muy similares, y la losa que influye en más del $30 \%$ del costo total del marco estructural, solo difiere entre sí por \$ 23.14 (ver tabla 9). Por otro lado, se observa que el individuo 1 o de menor costo, tiene la losa más cara de todos los resultados, por lo que quizás se puedan obtener resultados más económicos. En las siguientes figuras se observan las diferencias entre las secciones y los armados de cada individuo las losas resultaron con peraltes de 13 centímetros a diferencia de los 18 centímetros de los modelos anteriores, siendo \$ 162 más económica por cada metro cuadrado de construcción. Lo anterior se debe a que las dimensiones de crujías son de menor longitud en comparación con las estructuras de concreto reforzado de 3 y 6 niveles. 

Leal, Juan Serrano

Tabla 9. Dimensiones y costos de las secciones de cada individuo

\begin{tabular}{|c|c|c|c|c|c|c|}
\hline & Individuo & 1 & 2 & 3 & 4 & 5 \\
\hline \multirow{7}{*}{$\begin{array}{l}\mathscr{U} \\
\stackrel{0}{0} \\
\stackrel{0}{0} \\
\tilde{n}\end{array}$} & Columna 1 & $55 \times 55$ & $50 \times 50$ & $55 \times 55$ & $55 \times 55$ & $55 \times 55$ \\
\hline & Columna 2 & $55 \times 55$ & $50 \times 50$ & $55 \times 55$ & $50 \times 50$ & $55 \times 55$ \\
\hline & Columna 3 & $45 \times 45$ & $45 \times 45$ & $45 \times 45$ & $50 \times 50$ & $50 \times 50$ \\
\hline & Trabe 1 & $40 \times 75$ & $40 \times 90$ & $40 \times 75$ & $45 \times 75$ & $35 \times 75$ \\
\hline & Trabe 2 & $40 \times 70$ & $35 \times 75$ & $40 \times 75$ & $35 \times 75$ & $35 \times 70$ \\
\hline & Trabe 3 & $30 \times 60$ & $30 \times 60$ & $30 \times 55$ & $30 \times 55$ & $30 \times 60$ \\
\hline & Losa & 13 & 13 & 13 & 13 & 13 \\
\hline \multirow{7}{*}{ 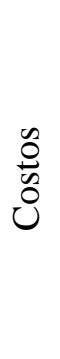 } & Columna 1 & $\$ 1,369.035$ & $\$ 1,419.91$ & $\$ 1,581.88$ & $\$ 1,369.035$ & $\$ 1,809.2$ \\
\hline & Columna 2 & $\$ 1,593.283$ & $\$ 1,318.436$ & $\$ 1,529.726$ & $\$ 1,318.436$ & $\$ 1,644.047$ \\
\hline & Columna 3 & $\$ 977.471$ & $\$ 970.657$ & $\$ 1,084.598$ & $\$ 1,318.436$ & $\$ 1,377.655$ \\
\hline & Trabe 1 & $\$ 1,561.693$ & $\$ 1,778.465$ & $\$ 1,561.693$ & $\$ 1,667.5$ & $\$ 1,562.171$ \\
\hline & Trabe 2 & $\$ 1,500.451$ & $\$ 1,377.91$ & $\$ 1,465.407$ & $\$ 1,492.632$ & $\$ 1,455.401$ \\
\hline & Trabe 3 & $\$ 996.02$ & $\$ 996.02$ & $\$ 960.79$ & $\$ 960.79$ & $\$ 1,003.97$ \\
\hline & Losa & $\$ 611.766$ & $\$ 611.766$ & $\$ 611.766$ & $\$ 611.766$ & $\$ 592.371$ \\
\hline
\end{tabular}

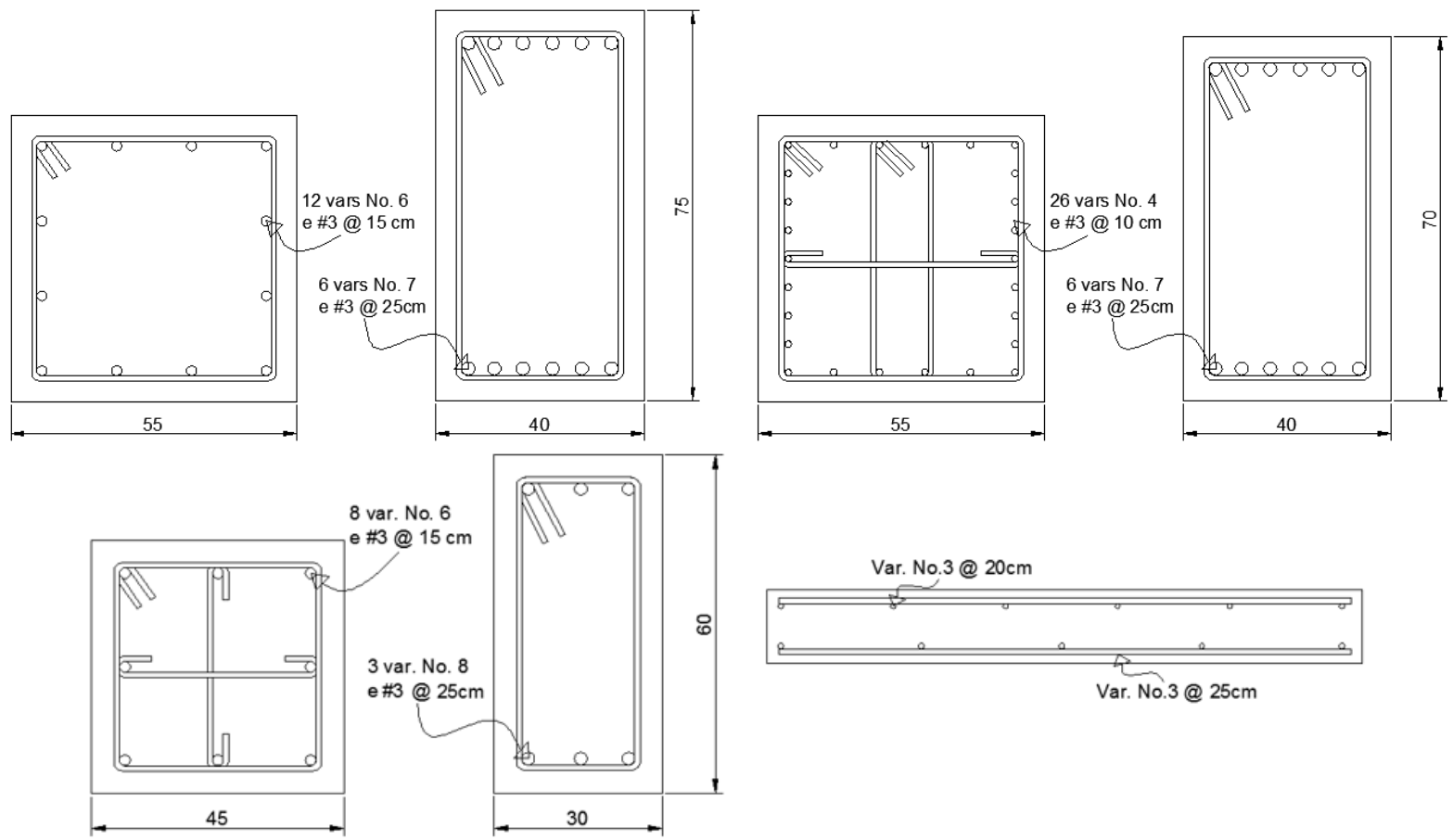

Figura 19. Armado de trabes, columnas y losa para el individuo 1 


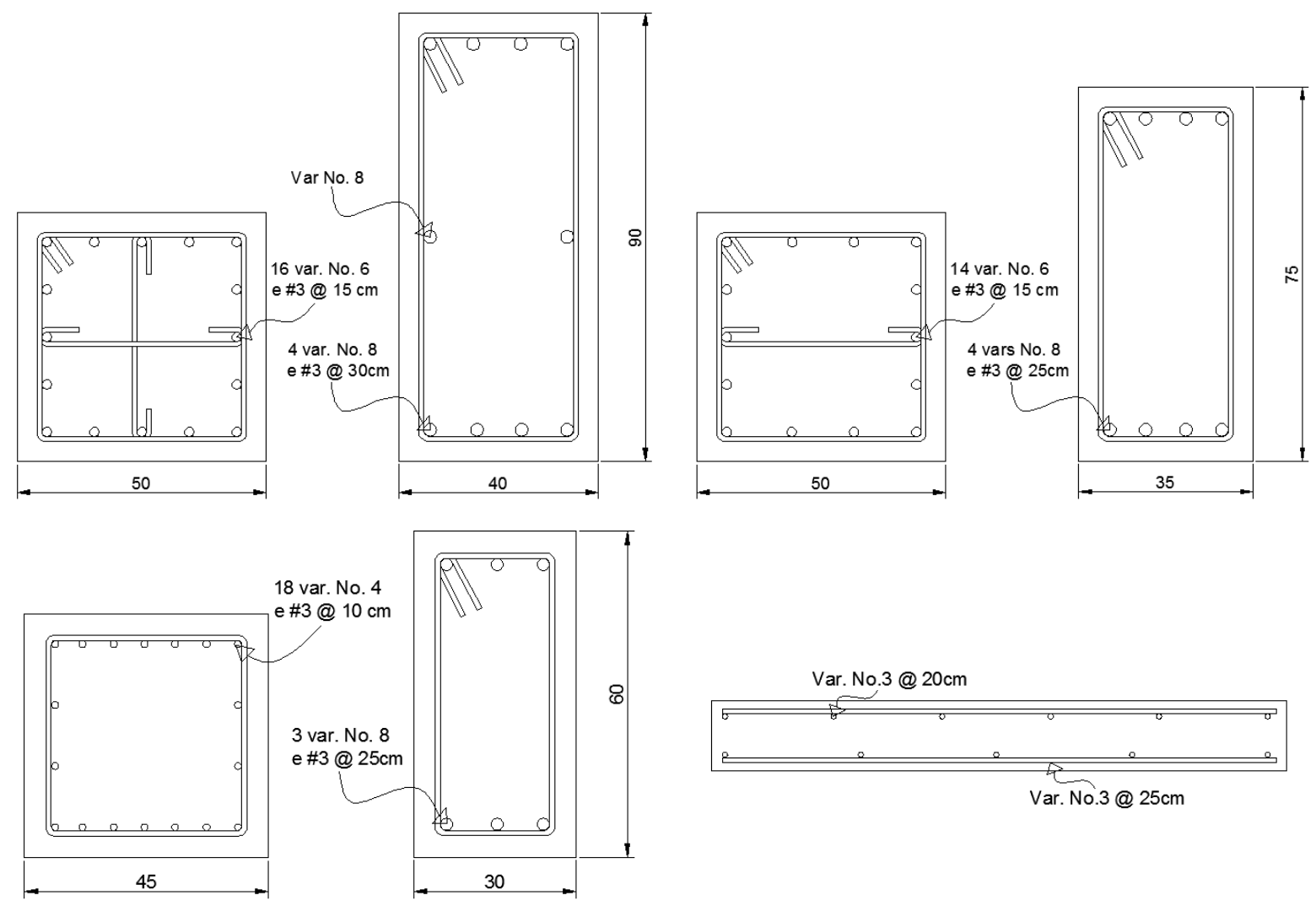

Figura 20. Armado de trabes, columnas y losa para el individuo 2

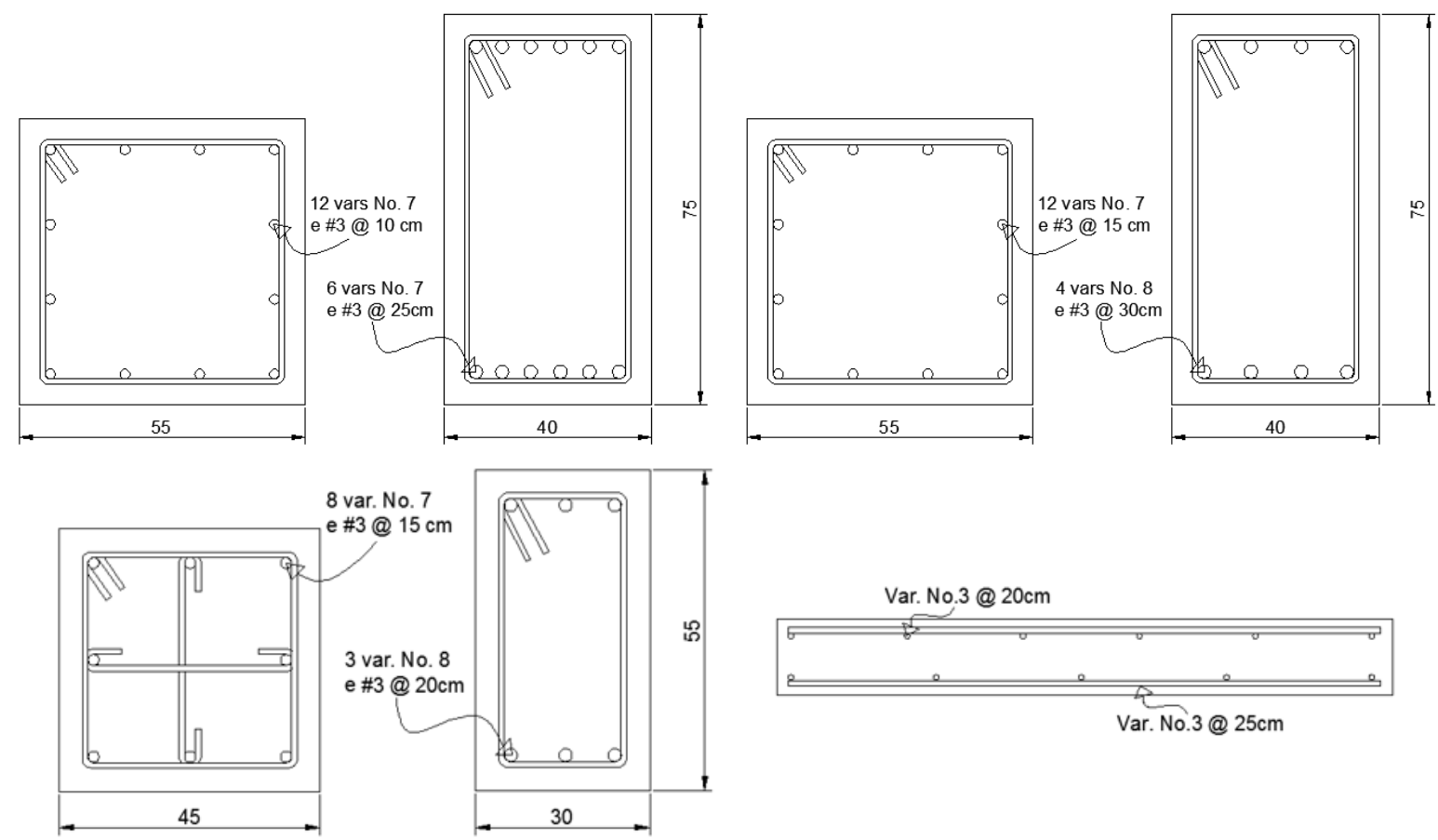

Figura 21. Armado de trabes, columnas y losa para el individuo 3 


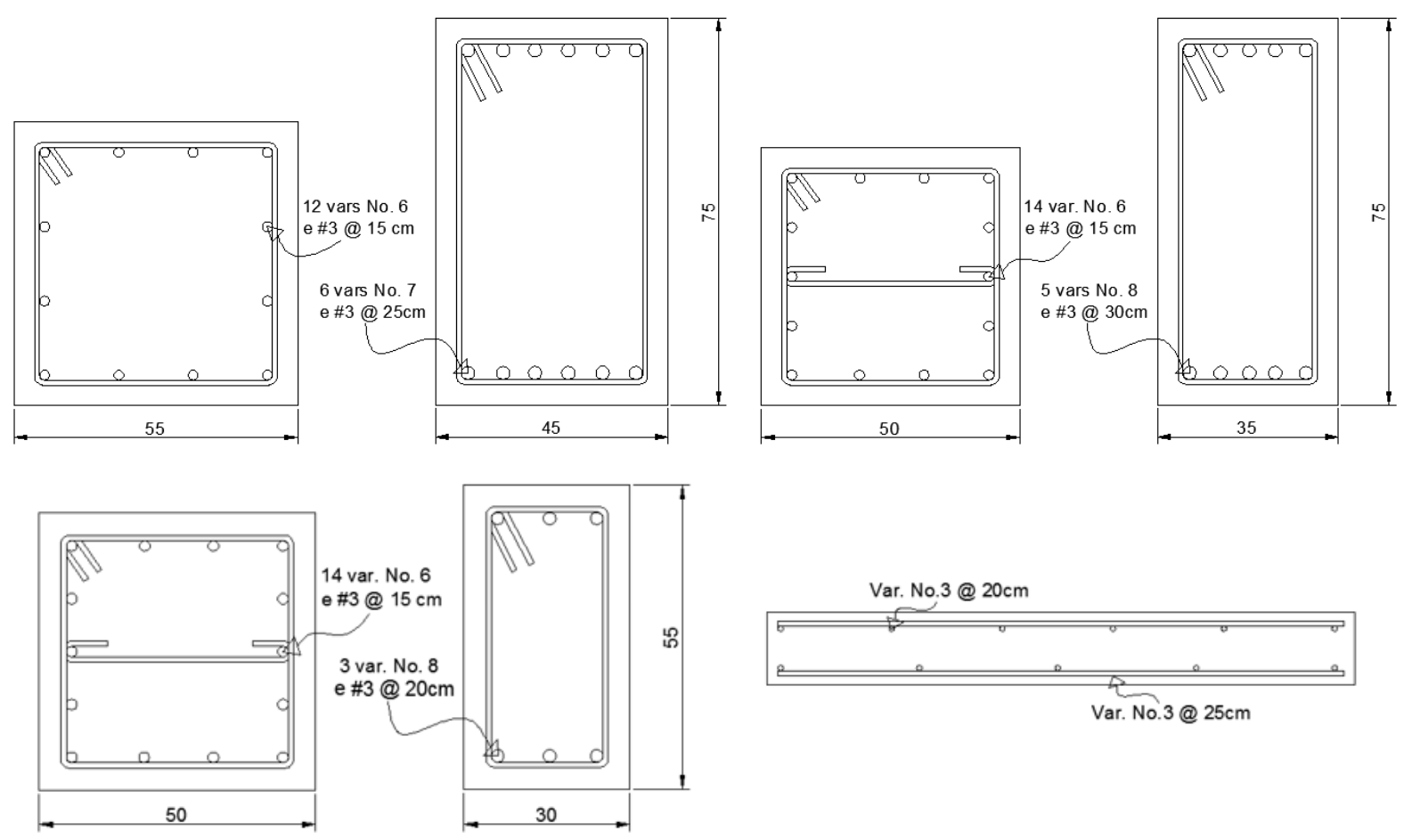

Figura 22. Armado de trabes, columnas y losa para el individuo 4
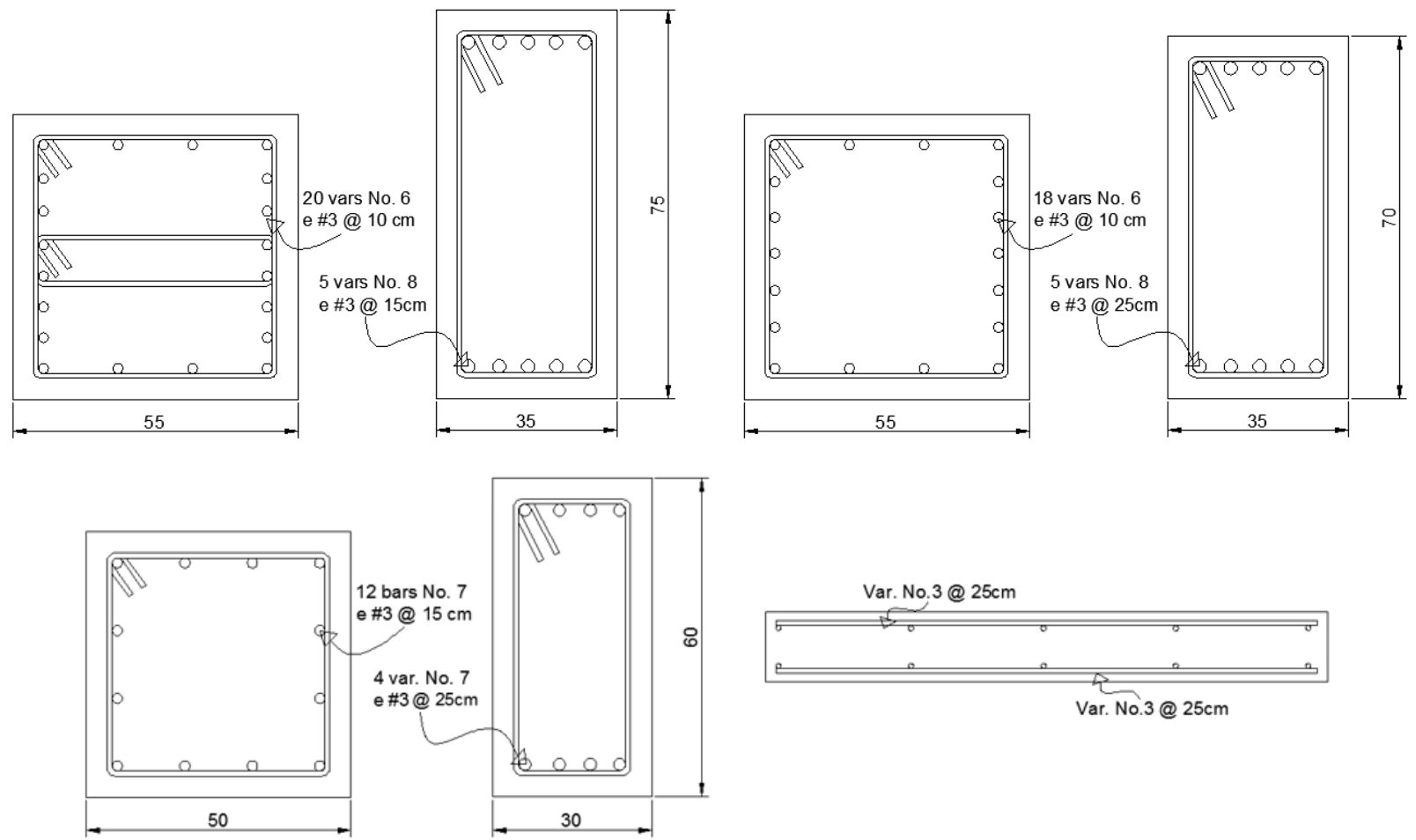

Figura 23. Armado de trabes, columnas y losa para el individuo 5 


\section{CONCLUSIONES}

La aplicación de algoritmos genéticos con optimización multi-objetivo mediante el método NSGAII resultó ser una excelente opción para el diseño de marcos de concreto reforzado tridimensionales. El comportamiento del software desarrollado por los autores fue el adecuado para cada modelo estudiado, con el paso de las generaciones las funciones objetivo disminuían, seleccionando cada vez secciones que daban lugar a una estructura que desarrollaba más su capacidad de deformación y resistencia y más económica. La función que depende de la distorsión $\left(F_{l}\right)$ ayudó para obtener secciones menos robustas y obtener distorsiones cercanas a 0.012 (límite sugerido por el RCDF); por otro lado, la función que depende del costo $\left(F_{2}\right)$ encontró las secciones más económicas sobre todo para losas. Además, las secciones que tenían una mayor influencia en el costo total del marco estructural (losas), resultaron ser muy similares para el conjunto de soluciones en los modelos CR-3 y CR-6; por lo tanto, se concluye que, para estos modelos, el número de individuos y de generaciones utilizadas fueron suficientes, caso contrario para el modelo CR-9, por lo que su Frontera de Pareto no está bien definida, aunque los resultados fueron satisfactorios. Para modelos en los que se busca obtener más de 5 elementos, se recomienda utilizar mayor número de individuos y de generaciones. Con el paso de las ejecuciones realizadas del programa de computadora se definía la Frontera de Pareto para cada modelo hasta obtener soluciones dominadas o similares a los resultados anteriores. Los marcos de CR más económicos de cada modelo resultaron con costos menores a $\$ 1,900 / \mathrm{m}^{2}$. Para el modelo CR-3 el costo fue de $\$ 1,499.94 / \mathrm{m}^{2}$, para el modelo CR-6 fue de $\$ 1,863.49 / \mathrm{m}^{2}$ y el modelo CR-9 fue de $\$$ $1,684.67 / \mathrm{m}^{2}$.

Por otro lado, la rigidez efectiva provocó grandes desplazamientos en los marcos, sobretodo en el modelo CR-6 donde las trabes de los niveles inferiores resultaron con peraltes de hasta $100 \mathrm{~cm}$ para mantener distorsiones menores a 0.012 , mientras que para los otros modelos la rigidez efectiva no afecto tanto por ser marcos de baja altura en el caso del modelo CR-3, o por tener crujías más cortas y por consecuencia losas con menor peralte y fuerzas sísmicas menores en el caso del modelo CR-9.

Con los resultados obtenidos se concluye que la combinación de la técnica multi-objetivo NSGA-II con el diseño sísmico de edificios de concreto provee una herramienta útil que facilita el diseño de estructuras, con las ventajas de obtener un conjunto de soluciones óptimas en un tiempo menor que el que se requiere en un diseño tradicional.

\section{AGRADECIMIENTOS}

Este trabajo se desarrolló con el apoyo económico brindado por el Consejo Nacional de Ciencia y Tecnología CONACYT a través de Proyectos Ciencia Básica y la beca otorgada a varios de los autores. Se agradece el apoyo de la Universidad Autónoma de Sinaloa dentro del proyecto PROFAPI 2015.

\section{REFERENCIAS}

Babaei, M y M Mollayi (2016), "Multi-objective optimization of reinforced concrete frames using NSGAII algorithm", Engineering Structures and Technologies, Vol. 8, No. 4, pp. 157-164. DOI: $\underline{10.3846 / 2029882 X .2016 .1250230}$

Back, B, T Laitinen y K Sere (1996), "Neural networks and genetic algorithms for bankruptcy predictions". Expert Systems with Applications, Vol. 11, No. 4, pp. 407-413. DOI:10.1016/S0957-417496000553 
Barraza, M (2013), “Algoritmos genéticos multi-objetivo y su empleo para el diseño sísmico óptimo de edificios de acero", Tesis de Maestría (Directores de Tesis: Edén Bojórquez y Eduardo Fernández), Universidad Autónoma de Sinaloa.

Barraza, M, E Bojórquez, E Fernández-González y A Reyes-Salazar (2017), "Multi-objective optimization of structural steel buildings under earthquake loads using NSGA-II and PSO", KSCE Journal of Civil Engineering. Vol. 21, pp. 488-500. DOI:10.1007/s12205-017-1488-7

Bojórquez, E, A Reyes-Salazar, S Ruiz y J Bojórquez (2013), “A new spectral shape-based record selection approach using $\mathrm{Np}$ and genetic algorithms", Mathematical Problems in Engineering. DOI: $\underline{10.1155 / 2013 / 679026}$

Cazacu, R y L Grama (2013), "Structural optimization with genetic algorithms and particle swarm optimization", Proceedings of the Annual Session of Scientific Papers IMT Oradea, Vol. 12, No. 22, pp. 19-22. DOI: 10.15660/AUOFMTE.2013-1.2778

Coello, C, F Hernández y F Farrera (1995), "Diseño óptimo de vigas de concreto reforzado mediante algoritmos genéticos". 2do. Congreso Internacional de Investigación en Ciencias Computacionales, Zacatepec, México.

Deb, K, S Agrawal, A Pratap y T Meyarivan (2000), "A fast elitist non-dominated sorting genetic algorithm for multi-objective optimization: NSGA-II", En International Conference on Parallel Problem Solving From Nature, Springer Berlin Heidelberg, September, pp. 849-858, DOI:10.1007/3-54045356-3_83

Deb, K y S Gulati (2001), "Design of truss-structures for minimum weight using genetic algorithms", Finite Elements Analysis and Design, Vol. 37, pp. 447-465. DOI:10.1016/S0168-874X(00)00057-3

Deb, K, A Pratap y T Meyarivan (2002), "A fast and elitist multiobjective genetic algorithm: NSGA-II”, IEEE Transactions on Evolutionary Computation, Vol. 6, pp. 182-197. DOI: 10.1109/4235.996017

Dede, T, S Bekiroglu y Y Ayvaz (2011), "Weight minimization of trusses with genetic algorithm", Applied Soft Computing, Vol. 11, pp. 2565-2575. DOI: 10.1016/j.asoc.2010.10.006

Erbatur, F, O Hasançebi, İ Tütüncü y H Kilic (2000), "Optimal design of planar and space structures with genetic algorithms", Computers and Structures, Vol. 75, pp. 209-224. DOI:10.1016/S00457949(99)00084-X

Goldberg, D (1989), Genetic algorithms in search, optimization, and machine learning, Addison-Wesley, Reading, MA.

Holland, J H (1975), Adaptation in natural and artificial systems. An introductory analysis with applications to biology, control and artificial intelligence, University of Michigan Press, Ann Arbor, Mich.

Kaveh, A, M Fahimi-Farzam y M Kalateh-Ahani (2015), "Performance-based multi-objective optimal design of steel frames structures: Nonlinear dynamic procedure", Scientia Iranica, Vol. 22, pp. 373387.

Koza, J R (1992), Genetic programming: on the programming of computer by means of natural selection, MIT Press.

Kripakaran, P, B Hall y A Gupta (2011), "A genetic algorithm for design of moment-resisting steel frames". Structural and Multidisciplinary Optimization”, Vol. 44, No. 4, pp. 559-574. DOI:10.1007/s00158011-0654-7

Kuri-Morales, A, y J Galaviz-Casas (2002), Algoritmos genéticos. Fondo de Cultura Económica/UNAM/IPN. 
Leps, M y M Sejnoha (2003), "New approach to optimization of reinforced concrete beams", Computers and Structures, Vol. 81, pp. 1957-1966. DOI: 10.1016/S0045-7949(03)00215-3

Leyva, H (2016), "Diseño de marcos de concreto reforzado con contraventeo restringido contra pandeo usando algoritmos genéticos", Tesis de Maestría (Directores de Tesis: Edén Bojórquez y Eduardo Fernández), Universidad Autónoma de Sinaloa.

Lohn, J D y S P Colombano (1999), “A circuit representation technique for automated circuit design”. IEEE Transactions on Evolutionary Computation, Vol. 3, pp. 205-219. DOI: 10.1109/4235.788491

Paulay, T y M J N Priestley (1992), Seismic design of reinforced concrete and masonry buildings, John Wiley and Sons, Inc.

Pezeshk, S, C Camp y D Chen (2000), "Design of nonlinear framed structures using genetic optimization", Journal of Structural Engineering, Vol. 126, No. 3, pp. 387-388. DOI:10.1061/(ASCE)07339445(2000)126:3(382)

Pique, J y M Burgos (2008), "Effective rigidity of reinforced concrete elements in seismic analysis and design", The 14th World Conference on Earthquake Engineering, pp. 12-17.

Prendes-Gero, M B y J M Drouet (2011), "Micro-scale truss optimization using genetic algorithm", Structural and Multidisciplinary Optimization, Vol. 43, pp. 647-656. DOI:10.1007/s00158-0100603-X

Prendes, M, A García y J Del Coz (2006), "Design optimization of 3D steel structures: genetic algotihms vs. classical techniques", Journal of Constructional Steel Research, Vol. 62, pp. 1303-1309. DOI: 10.1016/j.jcsr.2006.02.005

RCDF (2004), Reglamento de construcciones del Distrito Federal. Gaceta Oficial del Distrito Federal.

Reca, J y J Martínez (2005), "Genetic algorithms for the design of looped irrigation water distributions networks", Water Resources Research, Vol. 42, W05416. DOI:10.1029/2005WR004383

Rivera-Vargas, D, J Terrón y C Arce (2014), “Estimación de la rigidez agrietada para el análisis sísmico en estructuras de concreto reforzado", XIX Congreso Nacional de Ingeniería Estructural, SMIE.

Serrano, J (2016), "Optimizacion multi-objetivo de edificios de concreto reforzado mediante NSGA-II". Tesis de Maestría (Directores de Tesis: Edén Bojórquez y Eduardo Fernández), Universidad Autónoma de Sinaloa.

Da Silva, E (2001), “Otimização de estruturas de concreto armado utilizando algoritmos genéticos”, Tesis Doctoral, Universidad de Sao Paulo.

Túpac, Y (2011), “Algoritmos evolutivos en optimización multiobjetivos (MOEA)”, XII Congreso Internacional de Informática y Sistemas. 